\title{
Role of Candida albicans Tem1 in mitotic exit and cytokinesis
}

Stephen W. Milne, Jill Cheetham, Deborah Lloyd, Sophie Shaw, Karen Moore,

Konrad H. Paszkiewicz, Stephen J. Aves and Steven Bates *

College of Life and Environmental Sciences, University of Exeter, Exeter EX4 4QD, UK

* Corresponding author. E-mail: s.bates@ex.ac.uk; Tel. +44 1392 725174; Fax.+44 01392263434 


\section{ABSTRACT}

Candida albicans demonstrates three main growth morphologies: yeast, pseudohyphal and true hyphal forms. Cell separation is distinct in these morphological forms and the process of separation is closely linked to the completion of mitosis and cytokinesis. In Saccharomyces cerevisiae the small GTPase Tem1 is known to initiate the mitotic exit network, a signalling pathway involved in signalling the end of mitosis and initiating cytokinesis and cell separation. Here we have characterised the role of Tem1 in C. albicans, and demonstrate that it is essential for mitotic exit and cytokinesis, and that this essential function is signalled through the kinase Cdc15. Cells depleted of Tem1 displayed highly polarised growth but ultimately failed to both complete cytokinesis and re-enter the cell cycle following nuclear division. Consistent with its role in activating the mitotic exit network Tem1 localises to spindle pole bodies in a cell cycle-dependent manner. Ultimately, the mitotic exit network in C. albicans appears to co-ordinate the sequential processes of mitotic exit, cytokinesis and cell separation.

Keywords: mitotic exit; cytokinesis; cell cycle; cell separation; Tem1; Candida albicans 


\section{Introduction}

Candida albicans is an important opportunistic pathogen of humans, causing a wide range of clinical diseases ranging from common superficial infections to fatal systemic infections in the immunocompromised (Perlroth et al., 2007). A striking feature of $C$. albicans is its ability to grow in a variety of morphological forms, including yeast, pseudohyphal and true hyphal forms, depending on the growth conditions (Sudbery, 2011). It is widely accepted that the ability to switch forms is linked to virulence, as a number of mutants unable to switch morphologies demonstrate attenuated virulence (Braun et al., 2000; Lo et al., 1997; Saville et al., 2003; Zheng et al., 2004). The unicellular yeast form of C. albicans is reminiscent of budding yeast, displaying ovoid cells which separate after cytokinesis. Pseudohyphae also grow by budding but form chains of elongated cells which fail to separate and display constrictions at the septa. In contrast the true hyphal form consists of long narrow filaments with parallel sided walls that do not separate or display constrictions at the site of septation (Sudbery et al., 2004).

The cellular differences between the distinct morphological forms of $C$. albicans include the extent of polarised growth, nuclear migration, position of septation and ability to separate after cytokinesis (Sudbery et al., 2004). The control of these processes must be linked with the progression of the cell cycle (Berman, 2006; Whiteway and Bachewich, 2007). Cell separation is distinctive in the three main morphological forms, with yeast cells forming daughter cells that separate whereas pseudohyphal and hyphal cells remain attached. The process of cell separation is regulated by the RAM network (Regulation of Ace2 and Morphogenesis) (Saputo et al., 2012), whose activation is closely linked to the completion of mitosis and cytokinesis. In S. cerevisiae exit from mitosis and entry into cytokinesis is controlled by the Mitotic Exit Network (MEN) a GTPase regulated kinase cascade (reviewed in Bardin and Amon, 2001; de Bettignies and Johnston, 2003; McCollum and Gould, 2001; Segal, 2011). A similar cascade, the Septation Initiation Network (SIN), has been reported 
in Schizosaccharomyces pombe and Aspergillus nidulans, although in these organisms its primary role appears to be in regulating septation rather than mitotic exit (Bruno et al., 2001; Kim et al., 2006; Kim et al., 2009; Krapp et al., 2004).

The MEN in S. cerevisiae consists of the central GTPase Tem1, which is held inactive by the two component GAP (GTPase activating protein) Bub2 and Bfa1 and activated by Lte1.

Tem1 is asymmetrically localised to the daughter bound spindle pole body (SPB), where it is associated with Bub2/Bfa1 and held inactive (Bardin et al., 2000; Lee et al., 2001; Pereira et al., 2000). Whereas the GAPs are localised to the SPB the positive regulator Lte1 is restricted to the daughter cell cortex (Bardin et al., 2000; Jensen et al., 2002; Jensen et al., 2004) and activates Tem1 through controlling the localisation of Bfa1 and the spindle position checkpoint kinase Kin4 (Bertazzi et al., 2011; Chan and Amon, 2010; Falk et al., 2011; Geymonat et al., 2009). Overall this suggests the attractive spatial-temporal model where Tem1 is activated when the SPB reaches the daughter cell cortex, thus ensuring exit from mitosis only after correct genome partitioning. Following its activation Tem1 signals through a protein kinase cascade consisting of $\mathrm{Cdc} 15$ and Dbf2 with its associated factor Mob1 (Lee et al., 2001; Luca et al., 2001; Mah et al., 2001; Visintin and Amon, 2001). The final output of the MEN is the release of the phosphatase Cdc14 from the nucleolus, which then triggers mitotic exit through its dual action of promoting the degradation of B type cyclins and activating the cyclin dependant kinase inhibitor Sic1 (Shou et al., 1999; Visintin et al., 1998). In addition to signalling mitotic exit there is also considerable evidence that the MEN contributes to cytokinesis, with a number of its members re-localising to the bud neck at the point of cytokinesis (Hwa Lim et al., 2003; Menssen et al., 2001). Furthermore tem1, $c d c 15$ and mob1 mutants that bypass the mitotic exit defect all exhibit cytokinesis defects with a failure of actomyosin ring contraction (Lippincott et al., 2001; Menssen et al., 2001), and MEN mutants demonstrate synthetic lethality with IQG1 which is required for recruiting actin to the contractile ring (Corbett et al., 2006). Additionally phosphorylation of Hof1, which is required for cytokinesis, is MEN dependent (Corbett et al., 2006; Meitinger et al., 2011). 
Finally Tem 1 has also been reported to affect septin and actomyosin ring dynamics (Dobbelaere et al., 2003; Lippincott et al., 2001), and the release of Cdc14 is required for the nuclear localisation of the transcription factor Ace2 which regulates genes involved in septum degradation and cell separation (Brace et al., 2011; Weiss et al., 2002).

To date the only components of the MEN signalling cascade that have been analysed in $C$. albicans are the terminal kinase Dbf2 and phosphatase Cdc14 (Clemente-Blanco et al., 2006; Gonzalez-Novo et al., 2009). Interestingly in C. albicans, unlike S. cerevisiae, CDC14 is non-essential and is not sequestered into the nucleolus during the cell cycle. Instead Cdc14 is detected in the nucleus for the majority of the cell cycle and associates with SPBs during mitosis and finally at the bud neck for cytokinesis in yeast but not hyphal cells. (Clemente-Blanco et al., 2006) Deletion of CDC14 results in cells displaying a defect in cell separation; however, growth and morphology are not largely affected (Clemente-Blanco et al., 2006). In contrast to Cdc14, Dbf2 has been shown to be essential in C. albicans, and it plays a major role in spindle organisation and signalling for actomyosin ring contraction during cytokinesis (Gonzalez-Novo et al., 2009). These findings bring into question the regulation of mitotic exit and cytokinesis, and the function of the MEN, in C. albicans. In this work we demonstrate that the small GTPase Tem1 is essential for mitotic exit and cytokinesis in C. albicans. Tem1 depleted cells displayed highly polarised growth but ultimately failed to undergo septation and re-enter the cell cycle following nuclear division. Alongside the previous work on Cdc14 and Dbf2 this would suggest that the combined action of MEN signalling components in C. albicans is required to co-ordinate the processes of mitotic exit, cytokinesis and cell separation.

\section{Materials and methods}

\subsection{Strains, media and growth conditions.}

All strains constructed and used in the current study are listed in Table 1. C. albicans strains were grown at $30^{\circ} \mathrm{C}$ in either YPD medium (1\% yeast extract, $2 \%$ mycological peptone, $2 \%$ 
glucose) or SC medium ( $0.67 \%$ yeast nitrogen base, $2 \%$ glucose, $0.077 \%$ complete supplement mixture minus uracil) supplemented with $50 \mu \mathrm{g} / \mathrm{ml}$ uridine as required. For repression of the tetracycline-regulated promoter doxycyline was added to media at a final concentration of $20 \mu \mathrm{g} / \mathrm{ml}$. To select for nourseothricin resistance strains were grown on Sabouraud dextrose agar ( $1 \%$ mycological peptone, $4 \%$ glucose, $1.5 \%$ agar) containing 200 $\mu \mathrm{g} / \mathrm{ml}$ nourseothricin (Werner BioAgents, Jena, Germany). S. cerevisiae strains were grown in SC medium or SC+GAL $(0.67 \%$ yeast nitrogen base, $2 \%$ galactose, $0.077 \%$ complete supplement mixture minus uracil), and temperature sensitive strains were grown at $30^{\circ} \mathrm{C}$ and shifted to $37^{\circ} \mathrm{C}$ for phenotypic analysis. To score viability, cells from liquid cultures were washed in PBS, resuspended at a density of $1 \times 10^{6}$ cells $/ \mathrm{ml}$ and serial dilutions plated onto YPD medium.

\subsection{Strain construction.}

All strains constructed in this study (Table 1) were generated using PCR-mediated gene targeting strategies (Gerami-Nejad et al., 2001; Milne et al., 2011; Wilson et al., 2000; Wilson et al., 1999) and the oligonucleotides used are listed in Table S1. To regulate expression of CaTEM1 a strain containing a single copy of CaTEM1 under control of the tetracycline-regulated promoter was constructed. The first allele of CaTEM1 was disrupted using the URA3 recyclable PCR-mediated gene disruption system (Wilson et al., 2000), with the disruption cassette amplified from pDDB57, using primer pair TEM1-KO-F and TEM1KO-R, and transformed into C. albicans THE1 to generate a heterozygote strain. The URA3 marker was then recycled by selection on SC medium plus $2 \mathrm{mg} / \mathrm{ml} 5$-fluoroorotic acid and $50 \mu \mathrm{g} / \mathrm{ml}$ uridine. The coding sequence of the second copy of CaTEM1 was then placed under control of the TET promoter by promoter replacement with the URA3-TR promoter replacement cassette amplified from p99CAU1 (Nakayama et al., 2000), using primer pair TEM1-TET-F and TEM1-TET-R, and transformed into the heterozygote mutant to generate the TET-TEM1 strain. In order to follow Tem1 levels following promoter switch-off a V5- 
6xHis C-terminal tag was introduced into the copy under control of the TET promoter. The V5-6xHis-NAT1 cassette was amplified from pV5-NAT1 (Milne et al., 2011) using primer pair TEM1-V5-F and TEM1-NAT1-R and transformed into the TET-TEM1 strain with selection for nourseothricin resistance. Finally to overexpress CaCDC15 in the TET-TEM1 background the native promoter of one allele was replaced with the NAT1-ENO1p cassette (Milne et al., 2011) amplified with primer pairs CDC15-ENO-F1 and CDC15-ENO1-R.

For the C-terminal GFP tagging of Cdc3, Abp1 and Tub1 GFP-NAT1 tagging cassettes were amplified from pGFP-NAT1 (Milne et al., 2011), using primer pairs CDC3-GFP-F and CDC3NAT1-R, ABP1-GFP-F and ABP1-NAT1-R, and TUB1-GFP-F and TUB1-NAT1-R respectively, and transformed into the TET-TEM1 strain with selection for nourseothricin resistance. To GFP tag Tem1 at the C-terminus the GFP-URA3 tagging cassette (GeramiNejad et al., 2001) was amplified with primer pair TEM1-GFP-F and TEM-URA3-R and transformed into C. albicans CAI-4 (Fonzi and Irwin, 1993). For co-localisation studies Tub4 was RFP tagged in the TEM1-GFP background using the RFP-NAT1 cassette (Milne et al., 2011) and primer pair TUB4-RFP-F and TUB4-NAT1-R. Finally, TEM1-GFP was overexpressed through replacement of the native TEM1 promoter with the NAT1-ENO1p cassette (Milne et al., 2011) amplified with the TEM1-ENO1-F and TEM1-ENO1-R primers.

\subsection{Western blot analysis.}

Protein extracts were prepared in $100 \mathrm{mM}$ Tris-HCI pH 7.5, 0.01\% SDS, $1 \mathrm{mM}$ dithiothreitol, 10\% glycerol containing protease inhibitors (Roche Applied Science, Burgess Hill, UK) by glass bead disruption through four 20 second cycles at $6.5 \mathrm{~m} / \mathrm{s}$ in a FastPrep machine (Qbiogene, Cambridge, UK). Proteins $(50 \mu \mathrm{g})$ were then separated on a 10\% NuSep TriHEPES-SDS gel (Generon, Maidenhead, UK) and transferred to a polyvinylidene difluoride membrane. The membrane was probed with a mouse anti-V5 primary antibody (Invitrogen, Paisley, UK; 1:5000 dilution) followed by the anti-mouse IgG-horseradish peroxidase secondary antibody (Invitrogen, Paisley, UK; 1:10000 dilution) before proteins were detected 
using Lumiglo (New England Biolabs, Hitchin, UK) according to manufacturer's instructions. Ponceau S staining was used to confirm equal loading.

\subsection{Cell staining and microscopy.}

To visualise nuclei and septa $C$. albicans cells were fixed with $70 \%$ ethanol for 30 minutes followed by incubation in $1 \mu \mathrm{g} / \mathrm{ml}$ 4,6-diamidino-2-phenylindole dihydrochloride (DAPI) or 5 $\mu \mathrm{g} / \mathrm{ml}$ calcofluor white (CFW) respectively for $30 \mathrm{~min}$ and then washed five times with phosphate buffered saline (PBS). The actin cytoskeleton was visualised through staining ethanol fixed cells with $1 \mu \mathrm{g} / \mathrm{ml}$ rhodamine phalloidin for 30 minutes, and washing with PBS. To visualise GFP and RFP cells were grown in repressing or non-repressing conditions, briefly washed in PBS, and an aliquot of the cell suspension directly mounted on glass slides for microscopic examination. Epifluorescence and differential interference microscopy was carried out using a motorised inverted IX81 microscope (Olympus, Southend-on-Sea, UK) with epifluorescence illumination for GFP (Ex460/40, Em525/50), RFP/rhodamine (Ex562/40, Em624/40) and DAPI/CFW (Ex 400-440, Em 475 nm). Digital images were captured using a CoolSnap $\mathrm{HQ}^{2}$ camera (Roper Scientific, Germany) and processed using Metamorph software (version X; Molecular Devices, Wokingham, UK). Measurements were taken using the Image J software (http://rsbweb.nih.gov/ij/).

\subsection{Functional complementation of S. cerevisiae tem1-3 and yeast two hybrid analysis.}

The C. albicans and S. cerevisiae TEM1 open reading frames were PCR amplified using primer pairs CaTEM1-F/R and ScTEM1-F/R respectively and cloned into the S. cerevisiae expression vector pYES2.1 (Invitrogen, Paisley, UK). The resulting plasmids, pYESCaTEM1 and pYES-SCTEM1, and the negative control pYES-lacZ, were then transformed into the S. cerevisiae tem1-3 temperature sensitive mutant strain SSC237 (Corbett et al., 2006). The strains generated were then screened for temperature sensitivity at 30 and 37 ${ }^{\circ} \mathrm{C}$. For yeast two-hybrid analysis the C. albicans TEM1, BFA1 and BUB2 open reading frames were cloned into the bait pGBKT7 and prey pGADT7 vectors from the Matchmaker 
GAL4 two-hybrid system 3 (Clontech, France). All combinations of bait and prey vectors, plus controls of individual vectors, were then transformed into S. cerevisiae $\mathrm{AH} 109$ and interactions demonstrated through growth on SC media lacking Ade and His.

\subsection{RNA extraction and RNA-Seq analysis.}

For expression analysis triplicate RNA samples were generated from exponentially growing C. albicans yeast cells and from cells following $8 \mathrm{~h}$ inhibition of TEM1 expression. RNA extractions were performed by mechanical lysis and purified using the RNeasy mini kit (Qiagen, Crawley, UK), with optional DNase treatment, according to manufacturer's instructions. RNA integrity and quantity were checked on a 2100 Bioanalyzer (Agilent, Wokingham, UK) and sequencing libraries prepared using the TruSeq RNA sample preparation kit from Illumina (Little Chesterford, UK). Sequencing was carried out on an Illumina high throughput HiSeq 2000 system. Tophat2 (Trapnell et al., 2009) was used to perform remapping to the reference genome C. albicans SC5314, using the following parameters “--fusion-search --library-type fr-unstranded -I 10000 -r 50 --mate-std-dev 100 -p 8". Following this the Bedtools package (Quinlan and Hall, 2010) multicov module was used to extract counts for all reads overlapping with gene features in the reference genome.

Differential gene expression was then detected using DESeq software (Anders and Huber, 2010), and genes displaying a twofold change in expression and an adjusted $p$ value $<0.05$ were classed as differentially expressed. Gene expression data is available at the Gene Expression Omnibus (http://www.ncbi.nlm.nih.gov/geo/) accession number GSE48650.

\section{Results}

\subsection{Identification of a functional C. albicans TEM1 gene.}

A search of the C. albicans genome database (www.candidagenome.org) (Inglis et al., 2012) identified a single open reading frame (orf19.3001) previously annotated as CaTEM1 (Bachewich et al., 2005). CaTEM1 encodes a putative 200 amino acid protein which 
displays $46 \%$ identity ( $62 \%$ similarity) across the length of the protein to ScTem1, and contains all the G box GDP/GTP-binding motifs (Bourne et al., 1991; Wennerberg et al., 2005) expected in the G domain of a member of the Ras GTPase superfamily.

To determine if CaTEM1 is a true functional homolog of ScTEM1 we tested its ability to complement a S. cerevisiae tem1-3 temperature sensitive mutant. The Sctem1-3 mutant displays a clear phenotype, with cells growing at the permissive temperature of $30^{\circ} \mathrm{C}$ but failing to proliferate at the restrictive temperature of $37^{\circ} \mathrm{C}$ (Corbett et al., 2006). We cloned both SCTEM1 and CaTEM1 into the yeast expression vector pYES2.1 under control of the GAL1 promoter and transformed them, and the negative control pYES-lacZ, into the Sctem13 mutant. The resulting strains were then screened for temperature sensitive growth defects (Figure 1A). All strains grew equally well at $30^{\circ} \mathrm{C}$, however, the Sctem1-3 and Sctem1-3 (pYES-lacZ) strains failed to grow at $37^{\circ} \mathrm{C}$, as expected. Introduction of the wild type SCTEM1 or CaTEM1 genes into the Sctem1-3 mutant fully restored growth at the restrictive temperature. Furthermore this complementation was apparent even when the cells were grown under non-inducing as well as inducing conditions, suggesting that only a low level of expression of SCTEM1 or CaTEM1 is required for rescue. Therefore these results suggest that CaTEM1 is the true functional homolog of ScTEM1.

A search for other members of the MEN in the $C$. albicans genome identified clear potential homologs for all its major components including the putative bi-partite GAP Bfa1 (orf19.6080) and Bub2 (orf19.5827; (Bachewich et al., 2005; Finley et al., 2008)), the negative regulator Lte1 (orf19.2238), and the downstream signalling components Cdc15 (orf19.3545), Dbf2 (orf19.1223; (Gonzalez-Novo et al., 2009)), Mob1 (orf19.5528) and Cdc14 (orf19.4192; (Clemente-Blanco et al., 2006)), demonstrating that members of this pathway are present in C. albicans. In S. cerevisiae Tem1 is usually associated with ScBfa1 and ScBub2 for the majority of the cell cycle. To determine if the potential $C$. albicans homologs of Bfa1 and Bub2 associate with CaTem1 they were tested for interactions in the 
yeast two hybrid system. Introduction of the TEM1, BFA1 or BUB2 bait and prey vectors alone resulted in no activation of the HIS3 or ADE2 reporters (data not shown). All possible pair-wise combinations of bait and prey vectors were then screened for interactions. This analysis demonstrated that CaBfa1 and CaBub2 form a complex, and that CaTem1 interacts with this complex through CaBfa1 but not CaBub2 (Figure 1B). In addition it was seen that CaBfa1, CaBub2 and CaTem1 also interact with themselves in the yeast two hybrid system, perhaps suggesting the formation of a higher order structure or the potential for selfactivation of CaTem1. As far as we are aware the ability of Bfa1, Bub2 and Tem1 to selfassociate has not been reported in other systems, and it remains to be determined if this is a novel characteristic of the pathway components in C. albicans.

\subsection{CaTem1 localises to spindle pole bodies in a cell cycle regulated manner.}

To study the localisation of the Tem 1 protein in C. albicans we constructed a strain in which the TEM1 gene was C-terminally tagged with GFP under the control of its native promoter. In yeast, pseudohyphae and hyphae CaTem1-GFP was seen to localise to one or two intense spots reminiscent of a SPB localisation (Figure 2A), as expected from budding yeast. To confirm that CaTem1-GFP does localise to the SPB we tagged TUB4, which encodes the SPB-specific y-tubulin, with RFP in the CaTEM1-GFP background. Microscopic inspection of the resulting strain demonstrated a co-localisation of CaTem1-GFP with CaTub4-RFP, thus confirming the SPB localisation of CaTem1-GFP (Figure 2B). Further inspection of the CaTEM-GFP localisation revealed that its association with the SPB is cell cycle regulated (Figure 3A, B). Cells from an asynchronous culture were taken and the localisation of CaTEM-GFP scored in cells classed as either unbudded, or possessing a small or large bud. In unbudded cells, which are indicative of cells in the G1 phase of the cell cycle, no CaTem1-GFP signal was detected in the majority $(59 \%, n=64)$ of cells. However, in cells at the G1/S phase transition, characterised through the appearance of a small bud, one distinct CaTem1-GFP spot was present in $65 \%(n=57)$ of cells. Therefore CaTem1-GFP localises to 
the SPB early in the cell cycle, commensurate with the onset of S phase. Its appearance at this time broadly correlates with the expression profile of CaTEM1, which has been shown to start to increase in G1 and peak in the S phase of the cell cycle (Cote et al., 2009). During later stages of the cell cycle two CaTem1-GFP spots appeared following the duplication of the SPB, with one migrating into the daughter cell prior to mitosis. Within hyphal cells CaTem1-GFP also remains associated with the SPB. A single CaTem1-GFP spot appears in the mother cell prior to duplication and the subsequent migration of both into the germ tube where nuclear division occurs, in keeping with the nuclear dynamics seen in hyphal cells.

In S. cerevisiae some MEN components migrate to the bud neck during cytokinesis (Hwa Lim et al., 2003; Menssen et al., 2001), and in C. albicans both CaDbf2 and CaCdc14 relocalise to the bud neck at the end of mitosis (Clemente-Blanco et al., 2006; GonzalezNovo et al., 2009). However, CaTem1 under its native promoter was only seen associated with the SPB. To test this further we also over-expressed CaTEM1-GFP under control of the ENO1 promoter. However, no change in the localisation pattern of CaTem1-GFP was seen following over-expression (data not shown), suggesting that CaTem1-GFP may localise exclusively to the SPB from an early stage of the cell cycle.

\subsection{CaTEM1 is essential for vegetative growth.}

To investigate the function of CaTem1 in C. albicans we constructed a strain in which the single remaining copy of TEM1 was under the control of a tetracycline repressible promoter (Nakayama et al., 2000). The resulting strain, TET-TEM1, grew normally under nonrepressing conditions. However, under repressing conditions, through the addition of 20 $\mu \mathrm{g} / \mathrm{ml}$ doxycyline, the TET-TEM1 strain failed to form colonies (Figure 4A, B), therefore, demonstrating that TEM1 is essential in C. albicans. The failure to form colonies in the presence of doxycyline was also seen under conditions that induce hyphal growth (Figure 4C), indicating that TEM1 is essential for both yeast and hyphal development in C. albicans. 
As previously discussed $C$. albicans contains a clear homolog of the Cdc15 kinase that potentially acts downstream of Tem1. In both S. cerevisae and S. pombe overexpression of the native kinase, ScCdc15 and SpCdc7 respectively, rescues the phenotype associated with Tem1 depletion (Schmidt et al., 1997; Shirayama et al., 1994). Therefore, to determine if the essential function of Tem1 is also signalled through this kinase we screened for genetic suppression resulting from the overexpression of $C a C D C 15$. A strain was constructed where the native promoter of one allele of $C a C D C 15$ was replaced with the highly expressed ENO1 promoter in the TET-TEM1 background, and RT-PCR analysis confirmed a 15 fold increase in expression of $C D C 15$ in this strain. The overexpression of $C$ dc15 rescued the essential phenotype associated with TEM1 repression under both yeast and hyphal growth conditions (Figure 4B, C). Tem1 is therefore essential in C. albicans and its essential function is signalled through Cdc15.

\subsection{CaTem1 depleted cells form elongated filaments.}

In order to characterise the terminal phenotype of Tem1-depleted cells, growth and cell cycle progression were monitored following repression of TEM1 expression. After overnight growth in non-repressing conditions the TET-TEM1 strain was inoculated into repressing conditions and cells collected at different time points. Unlike Tem1 depleted cells in S. cerevisiae, which arrest as large budded cells, following $8 \mathrm{~h}$ repression in $C$. albicans the Tem1 depleted cells became filamentous (Figure 5A, B, C), with these filaments emerging exclusively from large budded cells. These mother cells were also significantly enlarged from an average width of $4.86+/-0.64 \mu \mathrm{m}$ following $4 \mathrm{~h}$ depletion to $5.96+/-1.04 \mu \mathrm{m}$ at $8 \mathrm{~h}$, compared to an average width of $4.61+/-0.41$ and $4.56+/-0.50 \mu \mathrm{m}$ at 4 and $8 \mathrm{~h}(\mathrm{n}=50)$ respectively for cells under non-repressing conditions. By $13 \mathrm{~h}$ repression all Tem 1 depleted cells had become filamentous, and these continued to elongate and form extensive filaments (Figure $5 \mathrm{~B}, \mathrm{C}$ ). This phenotype was ultimately cidal however as following $24 \mathrm{~h}$ growth in repressing conditions only $1.65 \%+/-1.40$ of TET-TEM1 cells retained viability compared to 
$96.67 \%+/-5.77$ of cells under non-repressing conditions, as determined through viable plate counts. Growth of the TET-TEM1 strain under non-repressing conditions was indistinguishable from the wild type strain grown in either repressing or non-repressing conditions, therefore indicating that the filamentous phenotype is due to loss of Tem1. To further confirm that the appearance of filamentous growth correlated with the depletion of Tem1 we epitope tagged Tem1 in the TET-TEM1 strain. Western blot analysis of Tem1-V5$6 x H i s$ levels showed repression following the addition of doxycyline with levels almost undetectable by $6 \mathrm{~h}$ (Figure 5D); therefore the appearance of filaments broadly correlates with a significant repression of Tem1. Overexpression of Cdc15, which we have shown rescues the essential phenotype associated with loss of Tem1, resulted in a complete restoration of yeast-like growth in cells depleted of Tem1, further supporting the essential function of Tem1 being signalled through Cdc15. The filaments formed following TEM1 repression lacked a constriction at their base, reminiscent of germ tubes, but were significantly swollen with an average width of $4.37+/-1.2 \mu \mathrm{m}(\mathrm{n}=40)$ at their appearance following $8 \mathrm{~h}$ repression. However following elongation the filaments became narrower with a width of $2.29+/-0.53 \mu \mathrm{m}$ and $2.20+/-0.45 \mu \mathrm{m}(\mathrm{n}=50)$ following 12 and $16 \mathrm{~h}$ repression respectively. Under the standard criteria used for defining hyphal growth (Sudbery et al., $2004)$ the majority of these filaments ( $82 \%$ and $92 \%$ at $12 \mathrm{~h}$ and $16 \mathrm{~h}$ respectively) would therefore be classed as true hyphae. True hyphal growth is associated with a hyper polarisation of the actin cytoskeleton. To monitor actin dynamics following TET-TEM1 repression we GFP tagged the actin binding protein Abp1 (Martin et al., 2007), which colocalises with the cortical actin cytoskeleton as defined by rhodamine phalloidin staining (Figure 6A). After $8 \mathrm{~h}$ repression, when wide filaments were forming, the Abp1-GFP protein localised to punctuate spots at the cell cortex as seen during yeast isotropic growth. However, at $12 \mathrm{~h}$ and $16 \mathrm{~h}$, when the filaments resembled hyphae, $50 \%$ and $80 \%$ of cells respectively displayed some accumulation of Abp1-GFP to the tip of the filament, although not as extensive as during true hyphal growth (Figure 6B, C). Finally true hyphal growth is 
dependent on the expression of the hyphal-specific cyclin HGC1, which is required to maintain polarised growth (Zheng et al., 2004). RT-PCR analysis of HGC1 expression demonstrated that it is expressed by the TET-TEM1 strain but only following $16 \mathrm{~h}$ repression (Figure 6D) when cells in the population ( 40\%) had typically started to loose viability. Taken together this would suggest that following TEM1 depletion the cells initially formed filaments, similar to that seen following treatment with hydroxyurea or deletion of the polo kinase Cdc5 (Bachewich et al., 2005; Bachewich et al., 2003), and at later time points these cells demonstrated further hyphal-like characteristics although ultimately they lost viability.

\subsection{CaTem1 is required for mitotic exit and cytokinesis.}

In S. cerevisiae Tem1 plays a central role in signalling mitotic exit and cytokinesis. To determine whether the filaments formed by Tem1-depleted cells have defects in nuclear division cells were fixed at various time points following repression and stained with DAPI. Control cells under non-repressing conditions underwent normal rounds of budding and division. However, the filaments formed following Tem1 depletion, even after extensive growth, typically contained two nuclei (Figure $7 \mathrm{~A}$ ). At 8 and $12 \mathrm{~h}$ following repression $74 \%$ $+/-4.9 \%$ and $70 \%+/-0.7 \%$ of filaments $(n=75)$ contained two nuclei with one present in the original mother cell and one in the filament. By 16 h $74 \%+/-0.7 \%(n=75)$ of cells still only contained two nuclei. However, at this point $26 \%+/-1.4 \%$ of cells contained both nuclei in the filament where the second resulted from migration of the nucleus from the original mother cell, leaving the mother cell lacking nuclear material and the filament binucleate.

To further characterise the cell cycle defect in Tem1-depleted cells we GFP tagged Tub1 in order to follow the mitotic spindle in these cells. The majority of cells demonstrating filamentous growth following 8 and 12 h repression of Tem 1 displayed an elongated mitotic spindle between the two DAPI stained bodies (Figure 7B). This would therefore suggest that cells depleted of Tem1, similar to S. cerevisiae, arrest in telophase and are unable to exit mitosis. Consistent with this finding RT-PCR analysis of the cyclin genes CCN1, CLB2 and 
CLB4 demonstrated a loss of expression of the G1 cyclin CCN1, and the continued expression of the mitotic cyclins CLB2 and CLB4 in cells depleted of Tem1 (Figure 7C). Overall this suggests that the Tem1-depleted cells undergo a single nuclear division, but then fail to exit mitosis and enter subsequent rounds of nuclear division.

The movement of the nucleus from the mother cell into the filament in cells depleted of Tem1 is suggestive of a cytokinesis and septation defect. To investigate this further the Tem1depleted cells were stained with calcofluor white. In all cells at 8, 12 and $16 \mathrm{~h}$ post repression only one chitin band was apparent at the original mother bud neck of the budded cell that gave rise to the filament (Figure 8). No chitin bands were seen in any of the filaments observed, indicating that these filaments are one continuous cellular compartment, and that Tem1-depleted cells have a cytokinesis and septation defect. To study this defect further we analysed septin dynamics by GFP tagging the Cdc3 septin. Septin rings are laid down early in the cell cycle and act as a scaffold to co-ordinate proteins required for cytokinesis. In Tem1-depleted cells septins were also only present at the original mother bud neck, where the chitin band was seen (Figure 9). No septin rings were present in the filaments formed, unlike in hyphal cells, being consistent with these cells displaying a block in the cell cycle. The septin ring seen at the original mother bud neck was also present in an hourglass collar structure which would suggest that the cells were arrested before the signal for septins to separate into two rings and for the actomyosin ring to assemble. In hyphal germ tubes a septin cap is initially present at the elongating tip (Sudbery et al., 2004). However, consistent with the filaments formed following Tem1 depletion not initially displaying hyphal-like characteristics, the septin cap was not present in the filaments resulting from Tem1 depletion. Altogether the defects in nuclear dynamics and septation in Tem1-depleted cells are in keeping with Tem1 playing an essential role in signalling mitotic exit and cytokinesis. 


\subsection{Global transcriptional analysis of Tem1 depleted cells.}

To determine the transcriptional profile resulting from Tem1 depletion we utilised RNASequencing (RNA-Seq). In order to avoid potential confounding issues resulting from the death of Tem1 depleted filaments we compared the transcriptional response at the early time point of $8 \mathrm{~h}$ repression, when filaments first started to appear, to an exponentially growing culture without repression. From this analysis we identified 527 genes which demonstrated a significant change in expression of two fold or greater with an adjusted $p$ value of $<0.05$. This gene set included 249 genes that were significantly down-regulated, ranging from 2 to 77 fold, and 278 significantly up-regulated genes demonstrating between 2 to 28 fold induction. The full list of genes displaying a significant change in expression is provided in Supplementary Tables S2 and S3. No enrichment for hyphal specific genes was detected in this gene set, consistent with filaments only displaying hyphal-like characteristics, and expressing HGC1, at later time points. However, the downregulated gene set was significantly enriched for genes whose expression is under cell cycle control. Cote et al. (2009) identified $7.8 \%$ (494/6350) of C. albicans genes as being cell cycleregulated, and $20.5 \%(51 / 249 ; p<0.0001)$ of the genes downregulated following TEM1 repression are present in this cell cycle-regulated gene set. These downregulated genes were also significantly enriched for genes whose expression peaks early in the cell cycle during the $\mathrm{G} 1 / \mathrm{S}$ or $\mathrm{S} / \mathrm{G} 2$ transition $(39 ; \mathrm{p}<0.0001)$. Of the ten downregulated genes whose expression is normally associated with the M/G1 transition six, including DSE1 and CHT3 which are required for cytokinesis and cell separation, are regulated through the RAM network by Ace2 (Kelly et al., 2004; Mulhern et al., 2006). The genes upregulated following Tem1 depletion show no overall enrichment for cell cycle regulated genes $(15 / 278 ; p=0.17)$, although those whose expression peaks in G1/S or S/G2 are significantly underrepresented $(4 ; p<0.01)$ and 11 of the 15 upregulated genes are normally associated with expression during $\mathrm{G} 2 / \mathrm{M}$ or M/G1. These results are consistent with Tem1-depleted cells undergoing a cell cycle block late in mitosis before the activation of the RAM network. 
In addition to cell cycle-regulated genes the downregulated gene set is also significantly enriched for Gene Ontology (GO) terms relating to glycolysis and associated categories, including monosaccharide catabolic process and glucose catabolic process. This enrichment, similar to that seen in cells deleted of Ace2 (Mulhern et al., 2006), appears to be glycolysis- not gluconeogenesis-specific as the glycolysis-specific genes PFK1, PFK2 and CDC19 are all downregulated, whereas the gluconeogenesis-specific genes, FBP1 and PCK1 were not significantly affected. The other major set of GO categories associated with Tem1 depletion is those associated with chromatin assembly and organisation, including the histones and NHP6A. This is also in keeping with Tem1-depleted cells undergoing a cell cycle blockade as the histones are known to be expressed during the S/G2 phase of the cell cycle (Cote et al., 2009), and a similar profile was reported in cells depleted of the polo kinase Cdc5 (Bachewich et al., 2005).

\section{Discussion}

Here we show that the small GTPase CaTem1 is a functional homolog of ScTem1 and is essential for signalling mitotic exit and cytokinesis in C. albicans. One of the most striking features following Tem1 depletion in C. albicans is the cells' forming highly elongated filaments. These filaments emerge from large budded cells and initially appear as wide nonparallel sided psuedohyphal-like cells, but at later time points taper down to resemble true hyphae. The promotion of filamentous growth is a common response by $C$. albicans to cell cycle arrest, with filamentation reported to occur following arrest at all stages of the cell cycle (Bachewich et al., 2005; Bachewich et al., 2003; Bachewich and Whiteway, 2005; Bai et al., 2002; Bensen et al., 2005; Berman, 2006; Chapa y Lazo et al., 2005; Chou et al., 2011). The precise morphology apparent however varies depending on the point of cell cycle arrest. For example when cells are blocked early in the cell cycle, such as following depletion of the $\mathrm{G}_{1}$ cyclin Cln3 (Bachewich and Whiteway, 2005; Chapa y Lazo et al., 2005), filaments form from unbudded cells. Alternatively, where arrest occurs later in the cell cycle, for example 
through depletion of the $\mathrm{G}_{2} / \mathrm{M}$ cyclin $\mathrm{Clb} 2$ (Bensen et al., 2005), filaments arise from budded cells. The appearance of filaments exclusively from large budded cells following the depletion of Tem1 is therefore consistent with these cells experiencing an arrest late in the cell cycle.

The filaments formed following Tem1 depletion were initially wide and did not demonstrate hyper-polarisation of the actin cytoskeleton. This is in contrast to cells depleted of the mitotic cyclin $\mathrm{Clb2}$, where polarisation of actin is maintained and filaments form as a result of the failure to switch from apical to isotropic growth (Bensen et al., 2005), and is consistent with Tem1 acting late in the cell cycle after the action of Clb2. However ultimately the filaments resulting from Tem1 depletion became narrower and demonstrated some hyper-polarisation of actin, suggesting the activation of polarised growth in these cells. In addition at later time points these filaments also expressed the hyphal-specific cyclin Hgc1 (Zheng et al., 2004). This is in keeping with the delayed expression of hyphal-specific genes previously reported in filaments formed following depletion of the polo-like kinase Cdc5 or the anaphasepromoting complex coactivator Cdc20 (Bachewich et al., 2005; Bachewich et al., 2003; Chou et al., 2011), which are both required for activating mitotic exit. Filaments formed following the depletion of Cdc5 (Bachewich et al., 2005) or dynein (Finley et al., 2008) have both been shown to be dependent on the spindle checkpoint protein Bub2. Here we have shown that Bub2 interacts with Tem1 through Bfa2 and presumably holds it inactive, further supporting the conclusion that Tem1-depleted cells form filaments as a result of a failure to exit mitosis. The filamentous growth phenotype demonstrated by Tem1-depleted cells however, similar to other cell cycle mutants, is terminal with these extensive filaments ultimately losing viability and failing to form colonies. The loss of viability seen is presumably due to defects associated with the arrest in telophase with a highly elongated spindle, potentially combined with the uncoupling of nuclear division and cellular growth seen in these cells affecting the nuclear cytoplasmic ratio. 
Nuclear dynamics and the presence of an elongated spindle in cells depleted of Tem1 are also in keeping with these cells failing to exit mitosis. The filaments formed predominantly contained two nuclear bodies, one in the original mother cell and one in the filament, connected by a long spindle. This would suggest that these cells arrest in telophase and fail to exit mitosis and enter subsequent rounds of nuclear division. Global transcriptional analysis of the Tem1 depleted cells is also in accordance with these cells arresting late in the cell cycle with a significant enrichment of G1/S and S/G2 specific genes in the downregulated gene set. Furthermore, genes controlled by the RAM network, which is activated by the MEN in S. cerevisiae, were also downregulated. This is in keeping with these cells arresting late in the cell cycle prior to the activation of the RAM network. At later time points following Tem1 depletion the nucleus in the original mother cell migrated into the filament, consistent with these cells also displaying a cytokinesis defect. A similar phenotype was reported following depletion of the mitotic cyclin Clb2 (Bensen et al., 2005), which is required for activating mitotic exit. Consistent with the failure to exit mitosis and activate cytokinesis septin and chitin bands were also not laid down in the filaments, and were only present at the original mother bud neck of the large budded cell that the filament emerged from. In addition the septin ring at the original mother bud neck was limited to the hour glass collar structure and failed to separate into two rings. A similar phenotype was seen in S. cerevisiae where the mitotic exit network is required to signal for septins to separate (Cid et al., 2001; Lippincott et al., 2001), and would suggest that in C. albicans Tem1 is also required for signalling both mitotic exit and cytokinesis.

CaTem1 localised exclusively to the SPB from the onset of S phase in both yeast and hyphae. However, unlike S. cerevisiae, where ScTem1 becomes preferentially recruited to the daughter bound SPB (Molk et al., 2004), CaTem1 was observed to localise to both SPBs for the duration of the cell cycle. The Tem1 homolog in S. pombe is also present on both SPBs, and its regulation is achieved through the asymmetrical localisation of its two component GAP (Cerutti and Simanis, 1999). This is also in keeping with more recent work 
in S. cerevisiae demonstrating that Lte1 functions to activate mitotic exit through controlling the localisation of Bfa2 and Kin4 (Falk et al., 2011; Geymonat et al., 2009). Therefore, a number of spatial and temporal mechanisms are involved in regulating Tem1 activity, and these show some variation between species.

In S. cerevisiae MEN components relocalise to the bud neck during cytokinesis (Hwa Lim et al., 2003; Menssen et al., 2001) and in C. albicans both Dbf2 and Cdc14 also migrate to the bud neck following mitosis (Clemente-Blanco et al., 2006; Gonzalez-Novo et al., 2009). CaTem1 however remains exclusively localised to the SPB from an early stage in the cell cycle, suggesting its essential function is signalled through other factors. Indeed, similar to what was seen in S. cerevisiae and S. pombe (Schmidt et al., 1997; Shirayama et al., 1994), overexpression of $C D C 15$ rescued the essential phenotype of cells depleted of CaTem1, suggesting that Cdc15 is immediately downstream of CaTem1and signals its essential function. Previous work on Dbf2 and Cdc14 has brought into question the role of the MEN in C. albicans. Here we have shown that cells depleted of CaTem1 demonstrate distinct phenotypes compared to those reported for cells lacking either Dbf2 or Cdc14. For example, whilst CaTem1 depleted cells form filaments and arrest with an elongated spindle Dbf2 depleted cells form chains and fail to initiate actomyosin ring contraction (Gonzalez-Novo et al., 2009), whereas Cdc14 is non-essential and its primary role is in signalling cell separation in yeast cells (Clemente-Blanco et al., 2006). Together this would suggest that the MEN in C. albicans is not a simple linear signalling cascade acting through a common end point. Instead the individual components of the MEN must direct specific cellular activities that together function to coordinate the processes of mitotic exit, cytokinesis and cell separation in C. albicans. 


\section{Acknowledgements}

We thank Clive Price for the S. cerevisiae tem1 mutant SSC237. This work was supported by a UK Biotechnology and Biological Sciences Research Council (BBSRC) project grant (No. BB/F009232/1) to S.B., and S.M. received a BBSRC studentship.

\section{Appendix A. Supplementary material}

Tables S1, S2 and S3 are found in supplementary material.

\section{References}

Anders, S., Huber, W., 2010. Differential expression analysis for sequence count data. Genome Biol. 11, R106.

Bachewich, C., et al., 2005. Cell cycle arrest during S or M phase generates polarized growth via distinct signals in Candida albicans. Mol Microbiol. 57, 942-59.

Bachewich, C., et al., 2003. Depletion of a polo-like kinase in Candida albicans activates cyclase-dependent hyphal-like growth. Mol Biol Cell. 14, 2163-80.

Bachewich, C., Whiteway, M., 2005. Cyclin CIn3p links G1 progression to hyphal and pseudohyphal development in Candida albicans. Eukaryot Cell. 4, 95-102.

Bai, C., et al., 2002. Spindle assembly checkpoint component CaMad2p is indispensable for Candida albicans survival and virulence in mice. Mol Microbiol. 45, 31-44.

Bardin, A. J., Amon, A., 2001. Men and sin: what's the difference? Nat Rev Mol Cell Biol. 2, 815-26.

Bardin, A. J., et al., 2000. A mechanism for coupling exit from mitosis to partitioning of the nucleus. Cell. 102, 21-31.

Bensen, E. S., et al., 2005. The mitotic cyclins Clb2p and Clb4p affect morphogenesis in Candida albicans. Mol Biol Cell. 16, 3387-400.

Berman, J., 2006. Morphogenesis and cell cycle progression in Candida albicans. Curr Opin Microbiol. 9, 595-601. 
Bertazzi, D. T., et al., 2011. The cortical protein Lte1 promotes mitotic exit by inhibiting the spindle position checkpoint kinase Kin4. J Cell Biol. 193, 1033-48.

Bourne, H. R., et al., 1991. The GTPase superfamily: conserved structure and molecular mechanism. Nature. 349, 117-27.

Brace, J., et al., 2011. Mitotic exit control of the Saccharomyces cerevisiae Ndr/LATS kinase Cbk1 regulates daughter cell separation after cytokinesis. Mol Cell Biol. 31, 721-35.

Braun, B. R., et al., 2000. Identification and characterization of TUP1-regulated genes in Candida albicans. Genetics. 156, 31-44.

Bruno, K. S., et al., 2001. SEPH, a Cdc7p orthologue from Aspergillus nidulans, functions upstream of actin ring formation during cytokinesis. Mol Microbiol. 42, 3-12.

Cerutti, L., Simanis, V., 1999. Asymmetry of the spindle pole bodies and spg1p GAP segregation during mitosis in fission yeast. J Cell Sci. 112 ( Pt 14), 2313-21.

Chan, L. Y., Amon, A., 2010. Spindle position is coordinated with cell-cycle progression through establishment of mitotic exit-activating and -inhibitory zones. Mol Cell. 39, 444-54.

Chapa y Lazo, B., et al., 2005. The G1 cyclin Cln3 regulates morphogenesis in Candida albicans. Eukaryot Cell. 4, 90-4.

Chou, H., et al., 2011. Orthologues of the anaphase-promoting complex/cyclosome coactivators Cdc20p and Cdh1p are important for mitotic progression and morphogenesis in Candida albicans. Eukaryot Cell. 10, 696-709.

Cid, V. J., et al., 2001. Cell cycle control of septin ring dynamics in the budding yeast. Microbiology. 147, 1437-50.

Clemente-Blanco, A., et al., 2006. The Cdc14p phosphatase affects late cell-cycle events and morphogenesis in Candida albicans. J Cell Sci. 119, 1130-43.

Corbett, M., et al., 2006. IQGAP and mitotic exit network (MEN) proteins are required for cytokinesis and re-polarization of the actin cytoskeleton in the budding yeast, Saccharomyces cerevisiae. Eur J Cell Biol. 85, 1201-15. 
Cote, P., et al., 2009. Transcriptional analysis of the Candida albicans cell cycle. Mol Biol Cell. 20, 3363-73.

de Bettignies, G., Johnston, L. H., 2003. The mitotic exit network. Curr Biol. 13, R301. Dobbelaere, J., et al., 2003. Phosphorylation-dependent regulation of septin dynamics during the cell cycle. Dev Cell. 4, 345-57.

Falk, J. E., et al., 2011. Lte1 promotes mitotic exit by controlling the localization of the spindle position checkpoint kinase Kin4. Proc Natl Acad Sci U S A. 108, 12584-90.

Finley, K. R., et al., 2008. Dynein-dependent nuclear dynamics affect morphogenesis in Candida albicans by means of the Bub2p spindle checkpoint. J Cell Sci. 121, 466-76.

Fonzi, W. A., Irwin, M. Y., 1993. Isogenic strain construction and gene mapping in Candida albicans. Genetics. 134, 717-28.

Gerami-Nejad, M., et al., 2001. Cassettes for PCR-mediated construction of green, yellow, and cyan fluorescent protein fusions in Candida albicans. Yeast. 18, 859-64.

Geymonat, M., et al., 2009. Lte1 contributes to Bfa1 localization rather than stimulating nucleotide exchange by Tem1. J Cell Biol. 187, 497-511.

Gonzalez-Novo, A., et al., 2009. Dbf2 is essential for cytokinesis and correct mitotic spindle formation in Candida albicans. Mol Microbiol. 72, 1364-78.

Hwa Lim, H., et al., 2003. Inactivation of mitotic kinase triggers translocation of MEN components to mother-daughter neck in yeast. Mol Biol Cell. 14, 4734-43.

Inglis, D. O., et al., 2012. The Candida genome database incorporates multiple Candida species: multispecies search and analysis tools with curated gene and protein information for Candida albicans and Candida glabrata. Nucleic Acids Res. 40, D66774.

Jensen, S., et al., 2002. Spatial regulation of the guanine nucleotide exchange factor Lte1 in Saccharomyces cerevisiae. J Cell Sci. 115, 4977-91.

Jensen, S., et al., 2004. Temporal coupling of spindle disassembly and cytokinesis is disrupted by deletion of LTE1 in budding yeast. Cell Cycle. 3, 817-22. 
Kelly, M. T., et al., 2004. The Candida albicans CaACE2 gene affects morphogenesis, adherence and virulence. Mol Microbiol. 53, 969-83.

Kim, J. M., et al., 2006. Isolation of mutations that bypass the requirement of the septation initiation network for septum formation and conidiation in Aspergillus nidulans. Genetics. 173, 685-96.

Kim, J. M., et al., 2009. Timely septation requires SNAD-dependent spindle pole body localization of the septation initiation network components in the filamentous fungus Aspergillus nidulans. Mol Biol Cell. 20, 2874-84.

Krapp, A., et al., 2004. SIN and the art of splitting the fission yeast cell. Curr Biol. 14, R72230.

Lee, S. E., et al., 2001. Order of function of the budding-yeast mitotic exit-network proteins Tem1, Cdc15, Mob1, Dbf2, and Cdc5. Curr Biol. 11, 784-8.

Lippincott, J., et al., 2001. The Tem1 small GTPase controls actomyosin and septin dynamics during cytokinesis. J Cell Sci. 114, 1379-86.

Lo, H. J., et al., 1997. Nonfilamentous C. albicans mutants are avirulent. Cell. 90, 939-49.

Luca, F. C., et al., 2001. Saccharomyces cerevisiae Mob1p is required for cytokinesis and mitotic exit. Mol Cell Biol. 21, 6972-83.

Mah, A. S., et al., 2001. Protein kinase Cdc15 activates the Dbf2-Mob1 kinase complex. Proc Natl Acad Sci U S A. 98, 7325-30.

Martin, R., et al., 2007. Functional analysis of Candida albicans genes whose Saccharomyces cerevisiae homologues are involved in endocytosis. Yeast. 24, 51122.

McCollum, D., Gould, K. L., 2001. Timing is everything: regulation of mitotic exit and cytokinesis by the MEN and SIN. Trends Cell Biol. 11, 89-95.

Meitinger, F., et al., 2011. Phosphorylation-dependent regulation of the F-BAR protein Hof1 during cytokinesis. Genes Dev. 25, 875-88. 
Menssen, R., et al., 2001. Asymmetric spindle pole localization of yeast Cdc15 kinase links mitotic exit and cytokinesis. Curr Biol. 11, 345-50.

Milne, S. W., et al., 2011. Cassettes for PCR-mediated gene tagging in Candida albicans utilizing nourseothricin resistance. Yeast. 28, 833-41.

Molk, J. N., et al., 2004. The differential roles of budding yeast Tem1p, Cdc15p, and Bub2p protein dynamics in mitotic exit. Mol Biol Cell. 15, 1519-32.

Mulhern, S. M., et al., 2006. Candida albicans transcription factor Ace2 regulates metabolism and is required for filamentation in hypoxic conditions. Eukaryot Cell. 5, 2001-13.

Nakayama, H., et al., 2000. Tetracycline-regulatable system to tightly control gene expression in the pathogenic fungus Candida albicans. Infect Immun. 68, 6712-9.

Pereira, G., et al., 2000. The Bub2p spindle checkpoint links nuclear migration with mitotic exit. Mol Cell. 6, 1-10.

Perlroth, J., et al., 2007. Nosocomial fungal infections: epidemiology, diagnosis, and treatment. Med Mycol. 45, 321-46.

Quinlan, A. R., Hall, I. M., 2010. BEDTools: a flexible suite of utilities for comparing genomic features. Bioinformatics. 26, 841-2.

Saputo, S., et al., 2012. The RAM network in pathogenic fungi. Eukaryot Cell. 11, 708-17.

Saville, S. P., et al., 2003. Engineered control of cell morphology in vivo reveals distinct roles for yeast and filamentous forms of Candida albicans during infection. Eukaryot Cell. $2,1053-60$.

Schmidt, S., et al., 1997. The Spg1p GTPase is an essential, dosage-dependent inducer of septum formation in Schizosaccharomyces pombe. Genes Dev. 11, 1519-34.

Segal, M., 2011. Mitotic exit control: a space and time odyssey. Curr Biol. 21, R857-9.

Shirayama, M., et al., 1994. The yeast TEM1 gene, which encodes a GTP-binding protein, is involved in termination of M phase. Mol Cell Biol. 14, 7476-82. 
Shou, W., et al., 1999. Exit from mitosis is triggered by Tem1-dependent release of the protein phosphatase Cdc14 from nucleolar RENT complex. Cell. 97, 233-44.

Sudbery, P., et al., 2004. The distinct morphogenic states of Candida albicans. Trends Microbiol. 12, 317-24.

Sudbery, P. E., 2011. Growth of Candida albicans hyphae. Nat Rev Microbiol. 9, 737-48.

Trapnell, C., et al., 2009. TopHat: discovering splice junctions with RNA-Seq. Bioinformatics. $25,1105-11$.

Visintin, R., Amon, A., 2001. Regulation of the mitotic exit protein kinases Cdc15 and Dbf2. Mol Biol Cell. 12, 2961-74.

Visintin, R., et al., 1998. The phosphatase Cdc14 triggers mitotic exit by reversal of Cdkdependent phosphorylation. Mol Cell. 2, 709-18.

Weiss, E. L., et al., 2002. The Saccharomyces cerevisiae Mob2p-Cbk1p kinase complex promotes polarized growth and acts with the mitotic exit network to facilitate daughter cell-specific localization of Ace2p transcription factor. J Cell Biol. 158, 885-900.

Wennerberg, K., et al., 2005. The Ras superfamily at a glance. J Cell Sci. 118, 843-6.

Whiteway, M., Bachewich, C., 2007. Morphogenesis in Candida albicans. Annu Rev Microbiol. 61, 529-53.

Wilson, R. B., et al., 2000. A recyclable Candida albicans URA3 cassette for PCR productdirected gene disruptions. Yeast. 16, 65-70.

Wilson, R. B., et al., 1999. Rapid hypothesis testing with Candida albicans through gene disruption with short homology regions. J Bacteriol. 181, 1868-74.

Zheng, X., et al., 2004. Hgc1, a novel hypha-specific G1 cyclin-related protein regulates Candida albicans hyphal morphogenesis. Embo J. 23, 1845-56. 
Table1. Strains used in this study

\begin{tabular}{|c|c|c|}
\hline Strain & Genotype & Reference \\
\hline \multicolumn{3}{|c|}{ C. albicans strains } \\
\hline \multirow[t]{2}{*}{ CAl-4 } & ura3s::imm434/ura3s:::imm434 & (Fonzi and \\
\hline & & Irwin, 1993) \\
\hline \multirow[t]{2}{*}{ THE1 } & 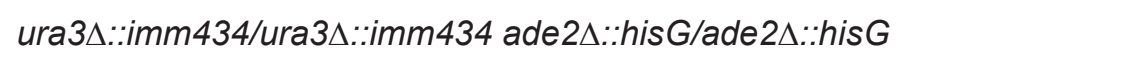 & (Nakayama \\
\hline & ENO1/eno1::ENO1-tetR-ScHAP4AD-3xHA-ADE2 & et al., 2000) \\
\hline SBC167 & As THE1 but TEM1/tem14::dp/200-URA3-dp/200 & This study \\
\hline SBC168 & As THE1 but TEM1/tem1 $1:: d p / 200$ & This study \\
\hline SBC169 & As THE1 but URA3-TETp-TEM1/tem14::dp/200 & This study \\
\hline SBC170 & As THE1 but URA3-TETp-TEM1-6xHis-V5-NAT1/tem14::dp/200 & This study \\
\hline \multirow[t]{2}{*}{ SBC171 } & As THE1 but URA3-TET1p-TEM1/tem1A::dp/200 CDC3-GFP- & This study \\
\hline & NAT1/CDC3 & \\
\hline SBC172 & As THE1 but URA3-TETp-TEM1/tem14::dp/200 ABP1-GFP-NAT1/ABP1 & This study \\
\hline SBC174 & As CAI4 but TEM1-GFP-URA3/TEM1 & This study \\
\hline SBC175 & As CAI4 but TEM1-GFP-URA3/TEM1 TUB4-RFP-NAT1/TUB4 & This study \\
\hline SBC176 & As CAI4 but NAT1-ENO1p-TEM1-GFP-URA3/TEM1 & This study \\
\hline \multirow[t]{2}{*}{ SBC177 } & As THE1 but URA3-TETp-TEM1/tem1A::dp/200 NAT1-ENO1p- & This study \\
\hline & $C D C 15 / C D C 15$ & \\
\hline SBC180 & As THE1 but URA3-TET1p-TEM1/tem1A::dp/200TUB1-GFP-NAT1/TUB1 & This study \\
\hline \multicolumn{3}{|c|}{ S. cerevisiae strains } \\
\hline \multirow[t]{2}{*}{ SSC237 } & MATa ade2-1 ura3-52 his3 leu2-3,-112 trp1-1 can1-100 tem1-3 & (Corbett et \\
\hline & & al., 2006) \\
\hline \multirow[t]{3}{*}{$\mathrm{AH} 109$} & 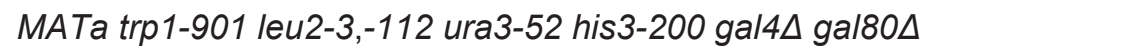 & (Clontech \\
\hline & LYS2::GAL1 $1_{\text {UAS }}-G A L 1_{\text {TATA }}-H I S 3$ MEL1 GAL2 UAS $-G A L 2_{\text {TATA }}-A D E 2$ & Laboratories \\
\hline & URA3::MEL1 $1_{\text {UAS }}-M E L 1_{\text {TATA-lacZ, MEL1 }}$ & Inc) \\
\hline
\end{tabular}




\section{Figure legends}

Fig. 1. CaTem1 functionally complements a $S$. cerevisiae tem 1 temperature sensitive mutant, and interacts with the CaBfa1 and Bub2 complex. (A) Strain SSC237 (Sctem1-3) was transformed with pYES-ScTEM1, pYES-CaTEM1 and the negative control pYES-lacZ, and grown in the presence of glucose at 30 and $37^{\circ} \mathrm{C}$. (B) Yeast two hybrid analysis of interactions between CaBfa1, Bub2 and Tem1. Growth on SD-W-L selects for markers on the bait and prey plasmids, whereas growth on the SD-W-L-A-H selective medium is indicative of an interaction.

Fig. 2. CaTem1-GFP localises to spindle pole bodies. (A) Strain SBC174 expressing CaTem1-GFP from its native promoter was grown as yeast (YEPD, $30^{\circ} \mathrm{C}$ ), pseudohyphae (YEPD, $37^{\circ} \mathrm{C}$ ) and true hyphae (YEPD $+20 \%$ Serum, $\left.37^{\circ} \mathrm{C}\right)$. (B) Exponentially growing yeast expressing CaTem1-GFP and CaTub4-RFP, from their native promoters, demonstrated co-localisation. Arrows indicate the position of CaTem1-GFP and CaTub4RFP. DIC and fluorescent micrographs were taken of unfixed cells. Bars, $10 \mu \mathrm{m}$.

Fig. 3. Localisation of CaTem1-GFP is cell cycle regulated. (A) Representative images of CaTem1-GFP localisation (indicated by arrows) in yeast cells at different stages of the cell cycle. Bars, $10 \mu \mathrm{m}$. (B) Quantification of the number of CaTem1-GFP foci present in unbudded $(n=64)$, small budded $(n=57)$ and large budded $(n=64)$ yeast cells.

Fig. 4. TEM1 is essential in C. albicans. (A) Growth of the TET-TEM1 strain on YPD medium without (TEM1 expression ON) and with $20 \mu \mathrm{g} / \mathrm{ml}$ doxycyline (TEM1 expression OFF). Overexpression of $C a C D C 15$ under control of the ENO1 promoter rescues the essential phenotype of TET-TEM1 following repression on doxycycline under yeast (B) and hyphal (C) growth conditions.

Fig. 5. Depletion of CaTem1 results in filament formation. Strain TET-TEM1 was grown under non-repressing $(\mathrm{A})$ or repressing $(\mathrm{B})$ conditions. Aliquots were taken at the time 
points indicated, fixed in ethanol, and viewed under differential interference microscopy. Bars, $10 \mu \mathrm{m}$. (C) Quantification of filament formation by TET-TEM1 cells following growth under repressing conditions. (D) Tem1-V5-6xHis protein levels following promoter shut-off. Strain SBC170 (TETp-TEM1-V5-6xHIS/tem1) was grown in repressing medium for 0, 2, 4, 6 and $8 \mathrm{~h}$, and samples assessed for Tem1-V5-6xHis protein levels by western blotting.

Fig. 6. Filaments formed following depletion of Tem1 ultimately display hyphal-like characteristics. (A) Abp1-GFP co-localises with the actin cytoskeleton as visualised through rhodamine phalloidin staining. (B) Abp1-GFP displays hyper-polarisation to the tip during hyphal growth. (C) Filaments formed following Tem1 depletion ultimately display an accumulation of Abp1-GFP at the tip of the filament. The TET-TEM1 strain, expressing Abp1-GFP, was grown under repressing conditions for the times indicated. Bars, $10 \mu \mathrm{m}$. (D) Filaments resulting from Tem1 depletion express the hyphal-specific cyclin HGC1 at late time points. RT-PCR analysis of HGC1 expression following growth of the TET-TEM1 strain under yeast $(\mathrm{Y})$ and hyphal $(\mathrm{H})$ growth conditions, and following Tem1 depletion for $0,4,8$, 12, 16 and $20 \mathrm{~h}$. EFB1 was used as an expression control.

Fig. 7. Tem1 depleted cells fail to exit mitosis. (A) The TET-TEM1 strain was grown in nonrepressing (YPD) or repressing conditions (YPD plus $20 \mu \mathrm{g} / \mathrm{ml}$ doxycyline) for the times indicated, fixed and strained with DAPI. (B) Tub1-GFP, and nuclei through DAPI staining. were visualised in the TET-TEM1 strain following 8 and $12 \mathrm{~h}$ of repression in YPD plus 20 $\mu \mathrm{g} / \mathrm{ml}$ doxycyline. Nuclei, following DAPI staining, are indicated by arrowheads. Bars, 10 um. (C) RT-PCR analysis of CCN1, CLB2 and CLB4 expression following growth of the TET-TEM1 strain following Tem1 depletion for 0, 8, 12, and $16 \mathrm{~h}$. EFB1 was used as an expression control.

Fig. 8. Septation is defective following Tem1 depletion. The TET-TEM1 strain was grown in non-repressing (YPD) or repressing conditions (YPD plus $20 \mu \mathrm{g} / \mathrm{ml}$ doxycyline) for the times indicated, fixed and strained with calcofluor white. Bars, $10 \mu \mathrm{m}$. 
Fig. 9. Septin dynamics are impaired following depletion of Tem1. The septin Cdc3, tagged with GFP, was visualised in the TET-TEM1 strain following $0,8,12$ and $16 \mathrm{~h}$ of repression in YPD plus $20 \mu \mathrm{g} / \mathrm{ml}$ doxycyline. Bars, $10 \mu \mathrm{m}$. 
A.

$30^{\circ} \mathrm{C}$

$$
\text { BY4741 }
$$

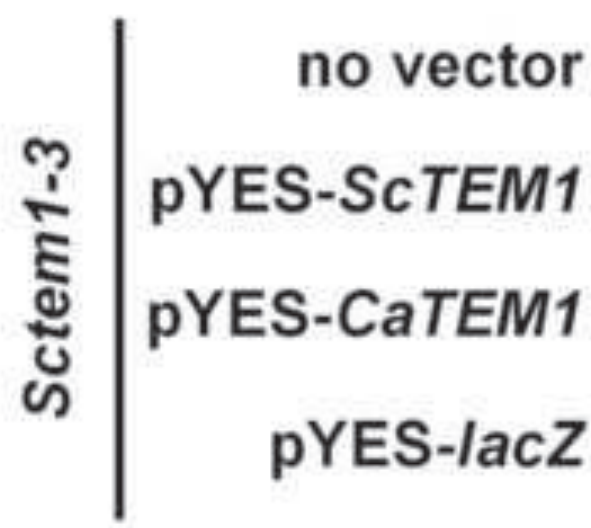

B.
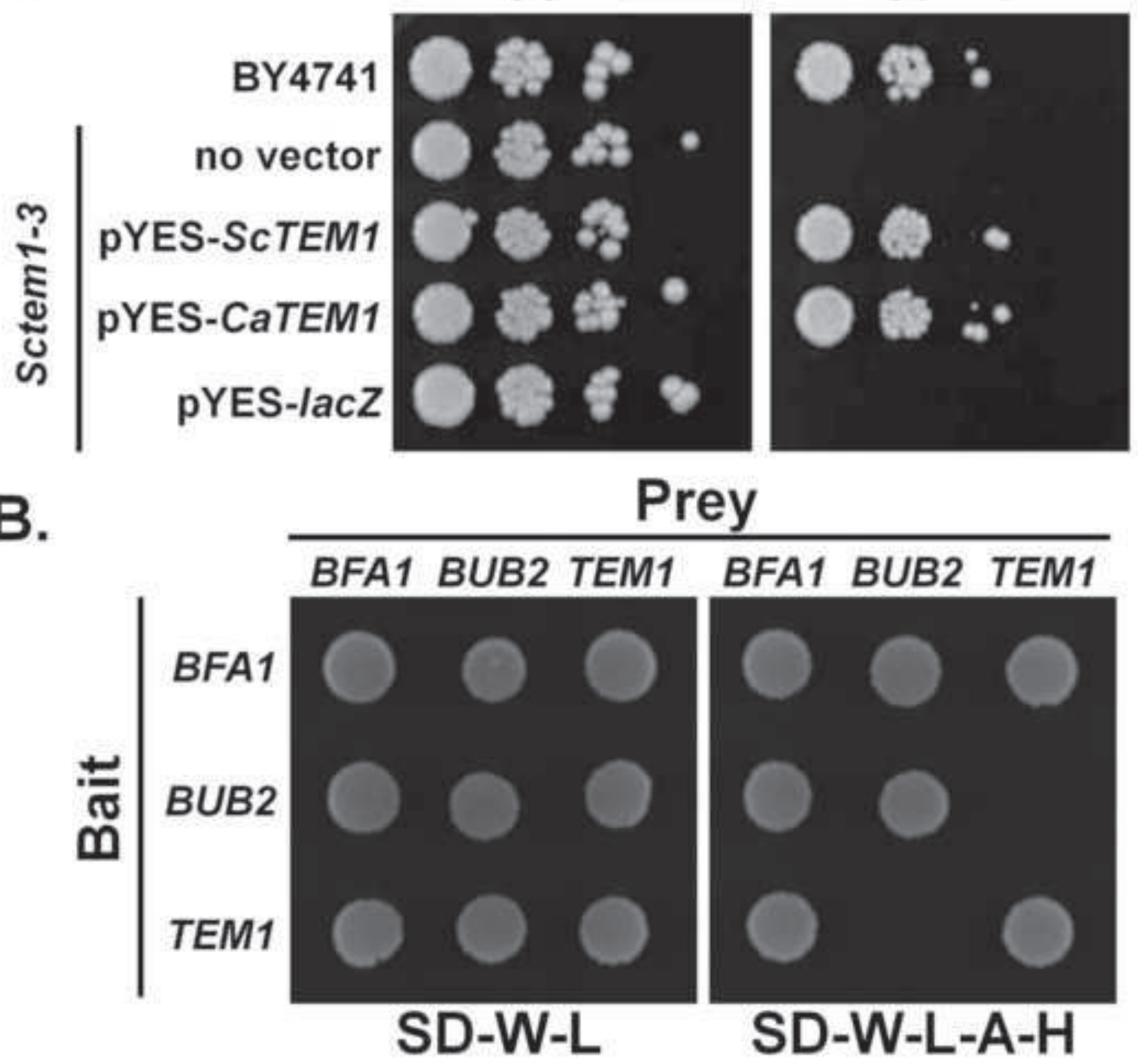

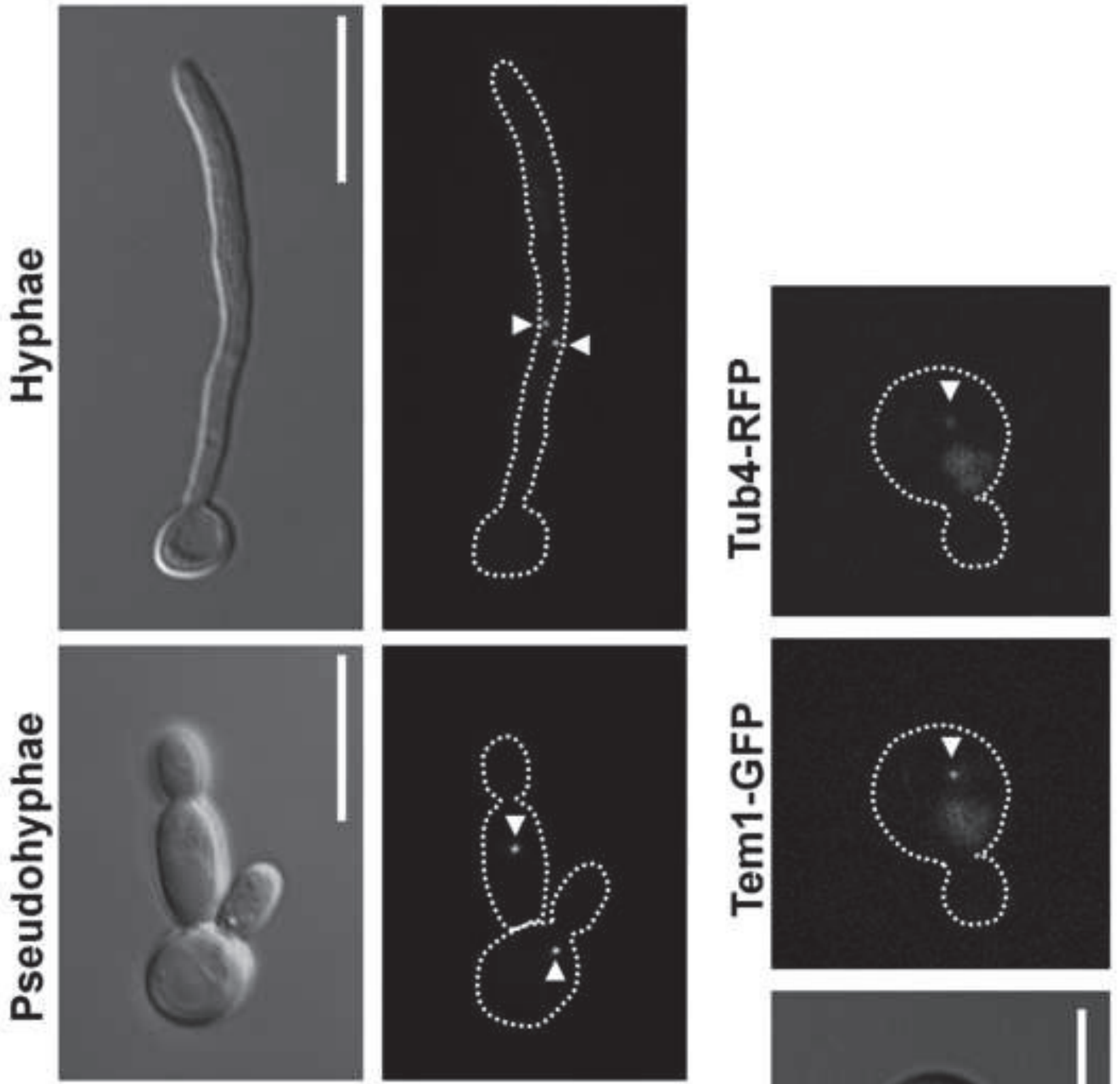

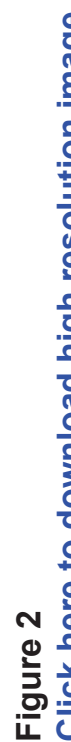

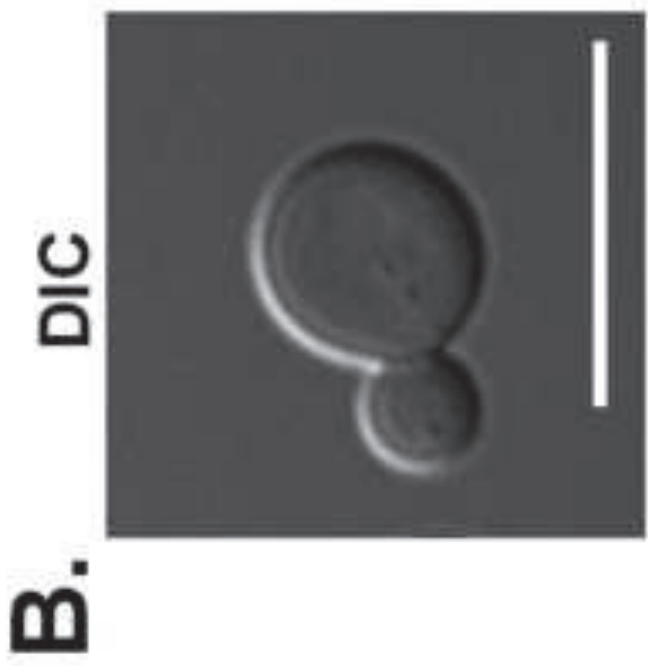




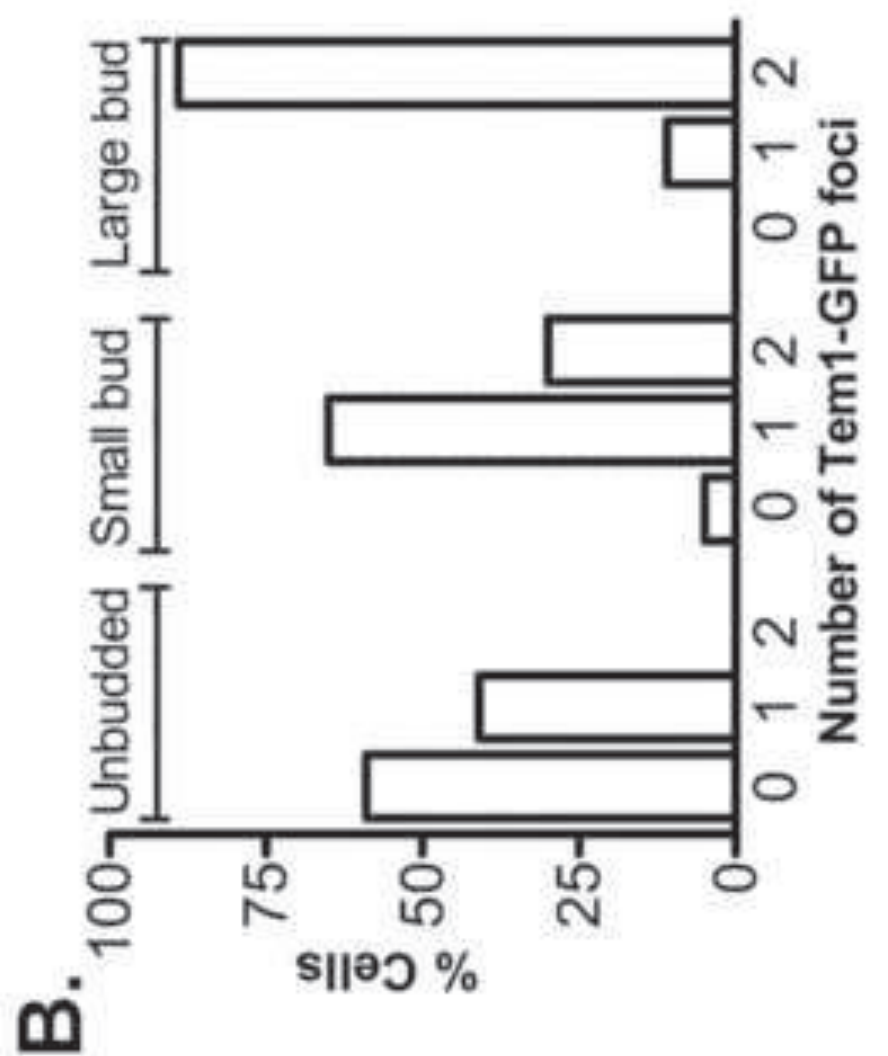

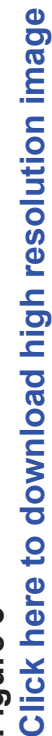
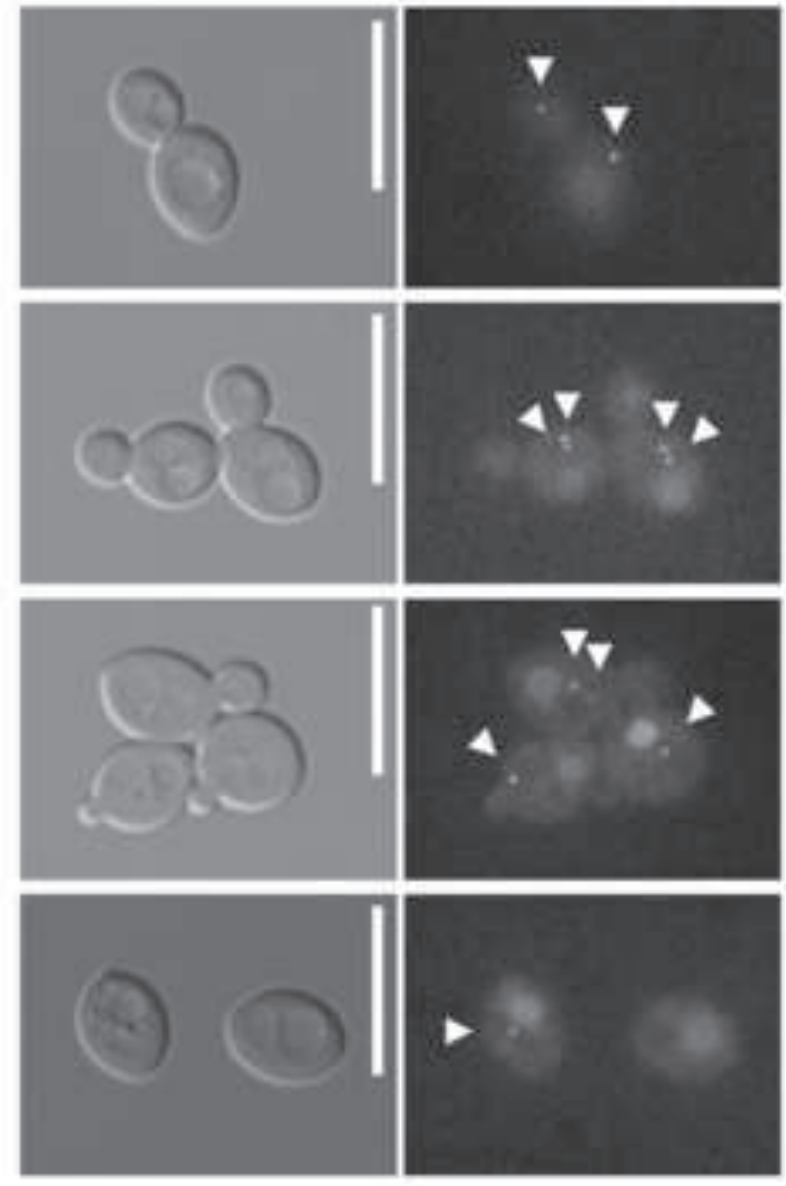

$<$ गา

m

응 
A.

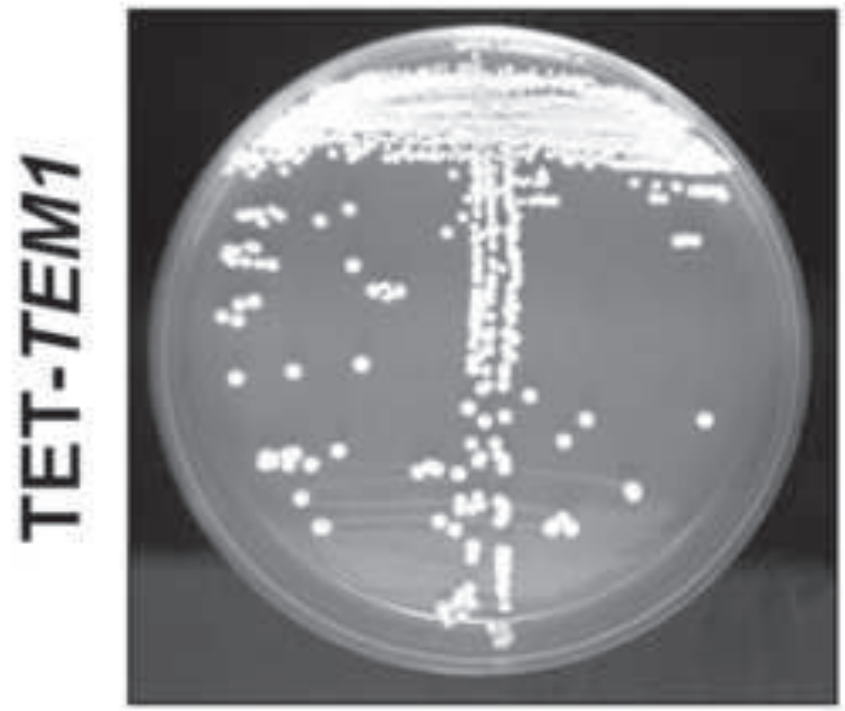

B.

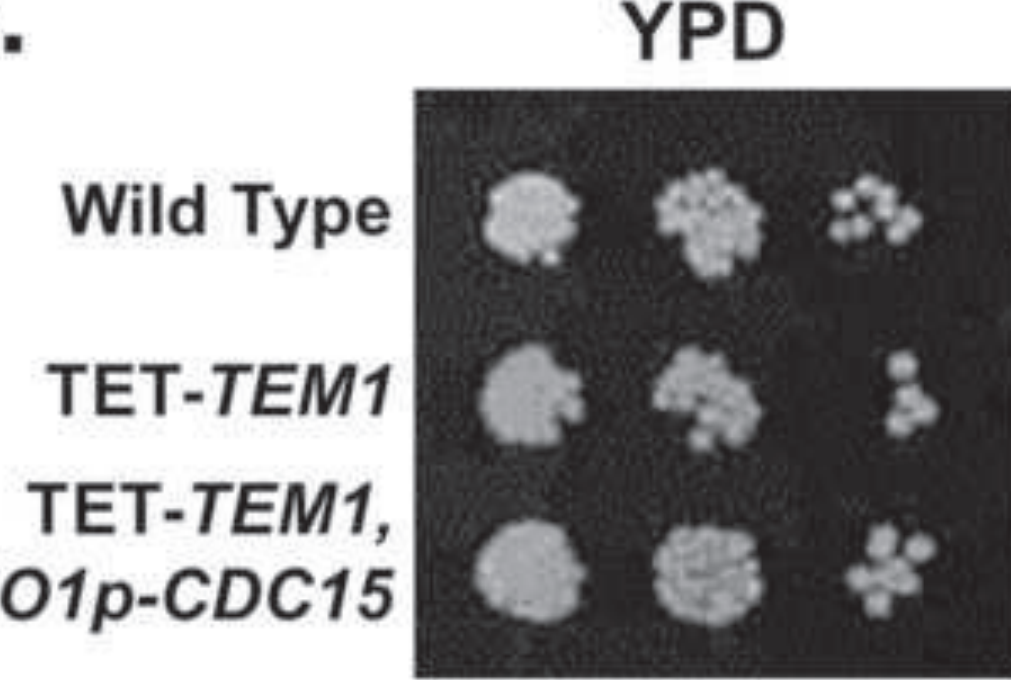

Serum

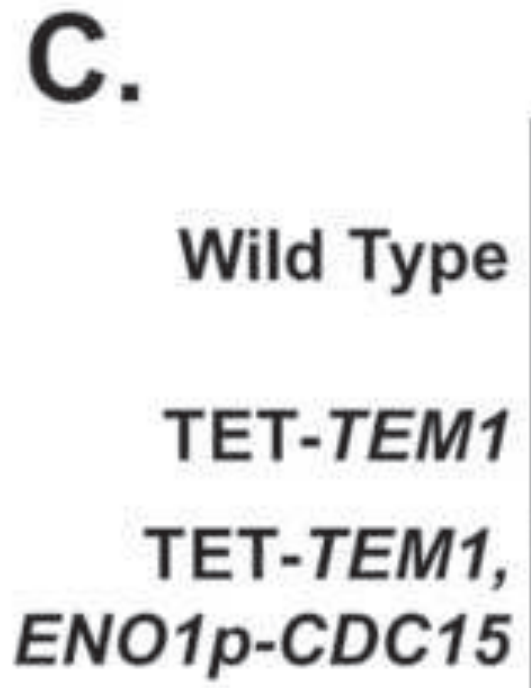

\section{YPD}

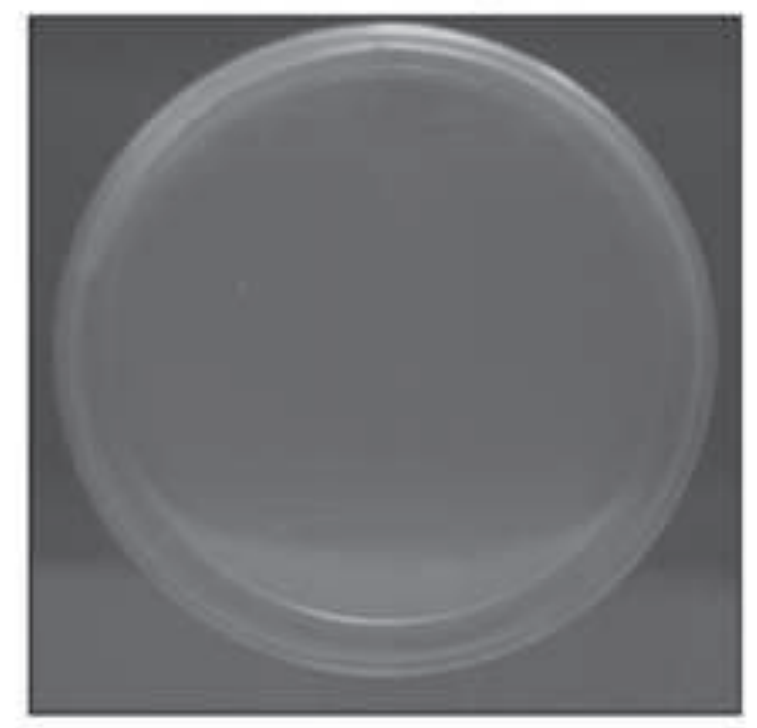

YPD+DOX

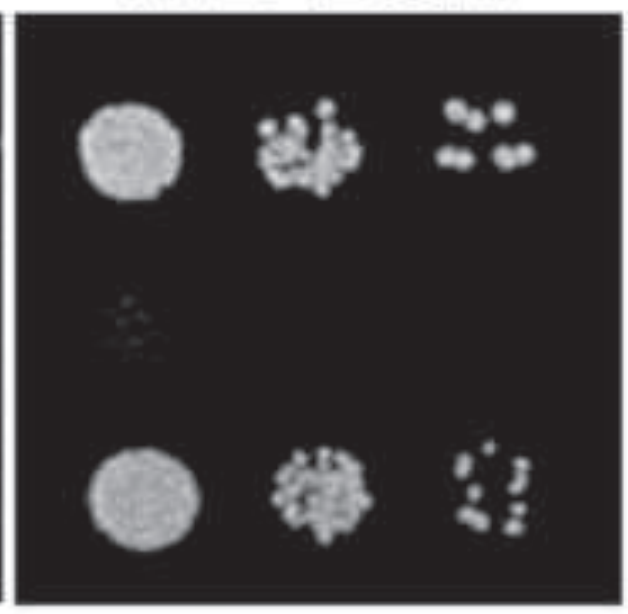

\section{Serum+DOX}

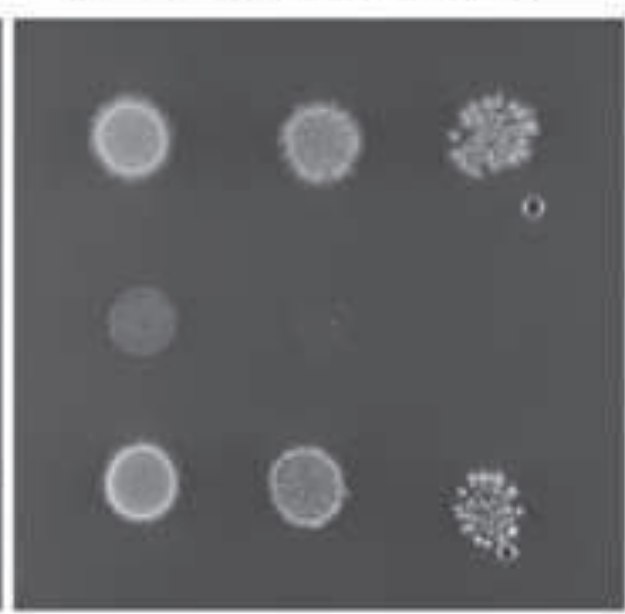


Click here to download high resolution image
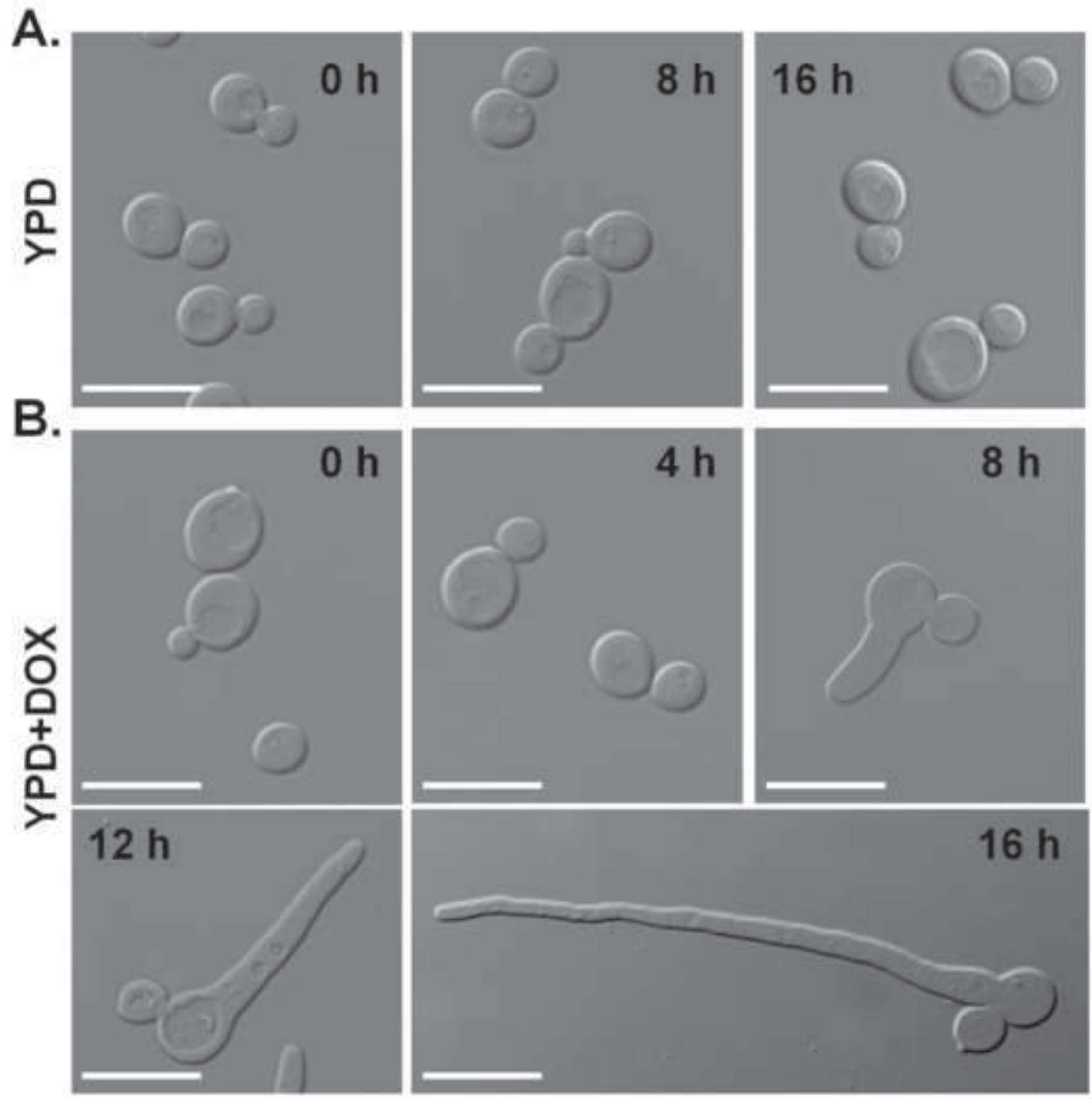

C.

D.
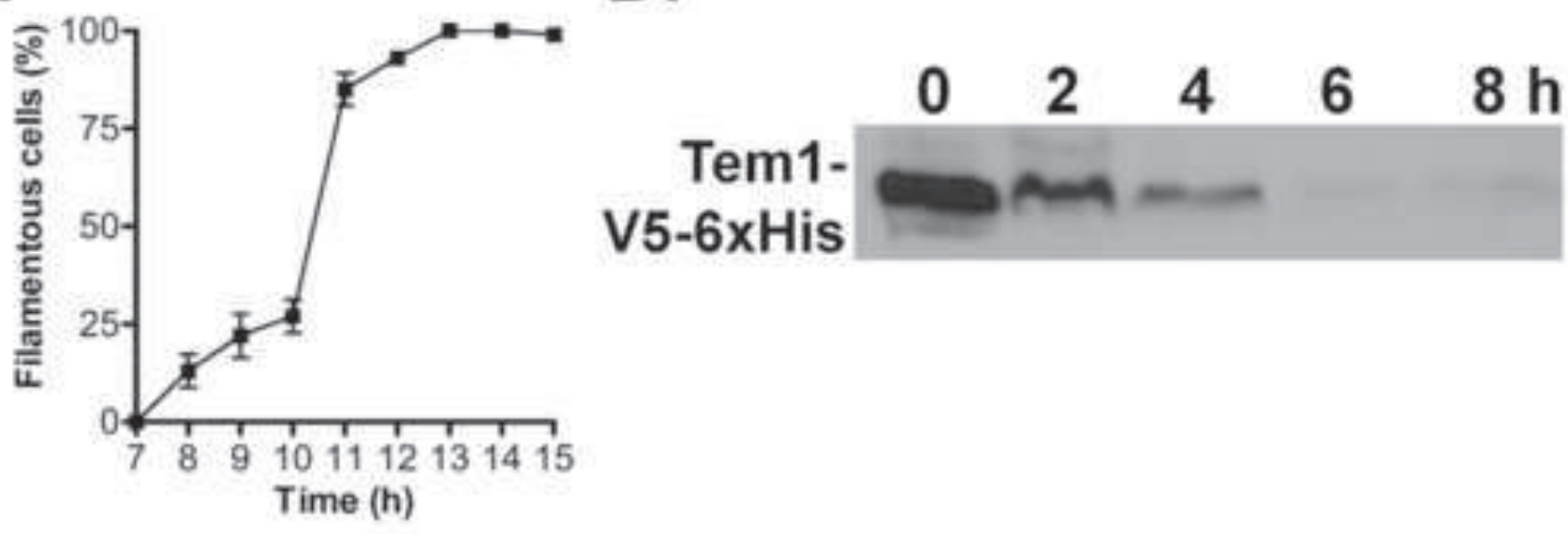
A.

DIC
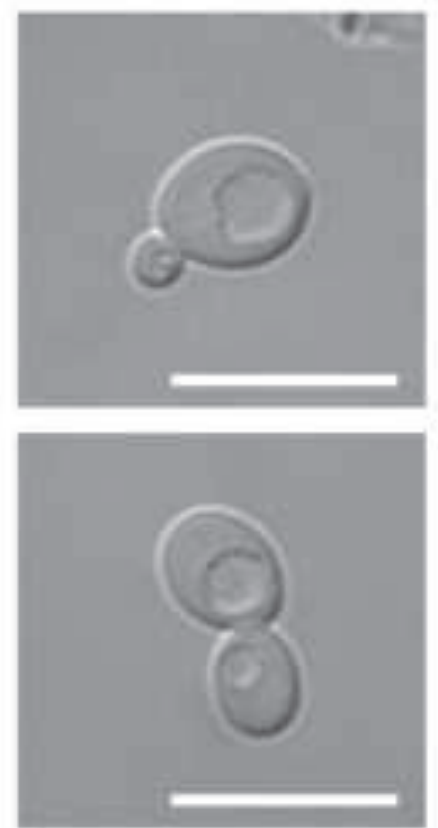

C.
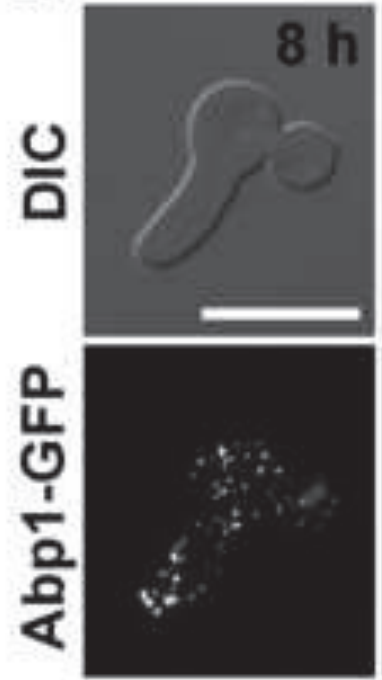

D.

Y

HGC1 EFB1
Abp1-GFP
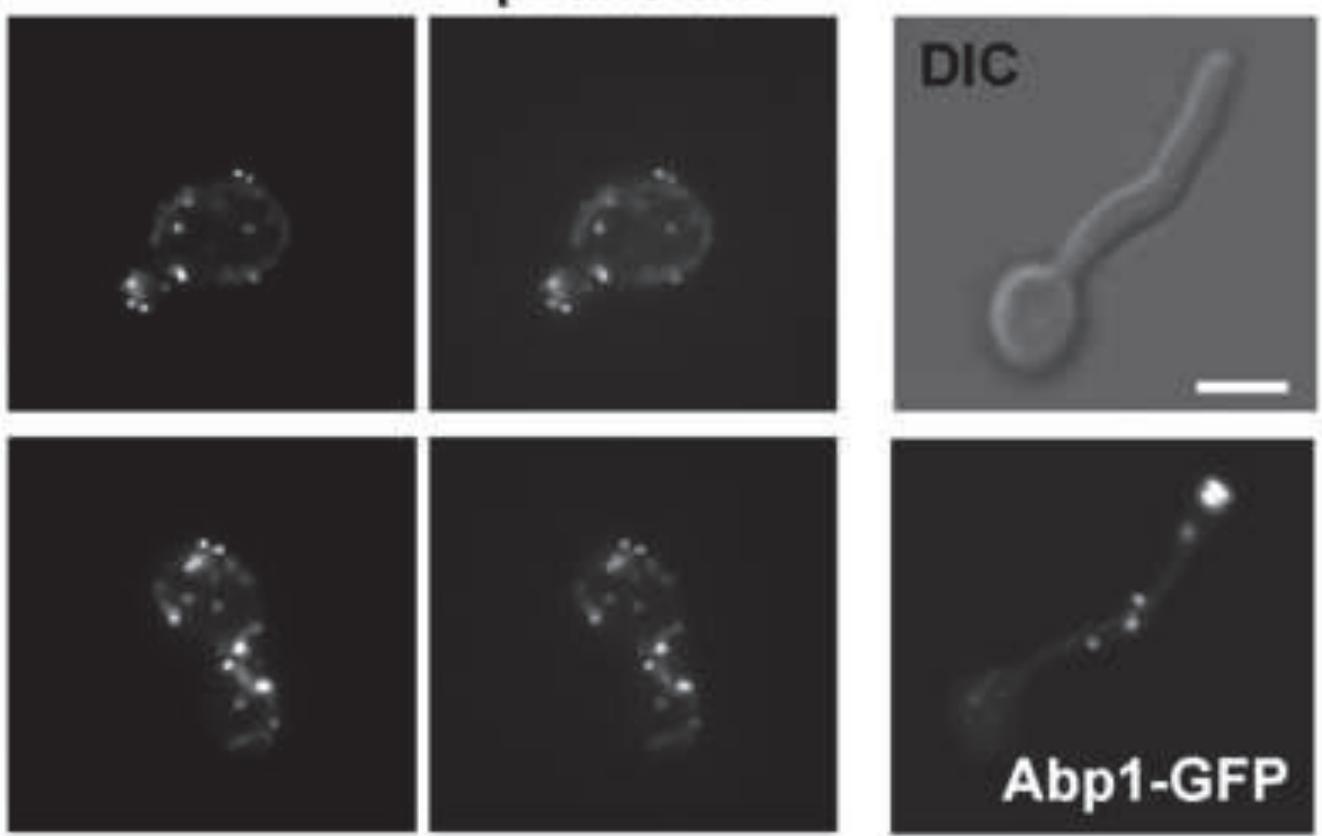

Abp1-GFP 

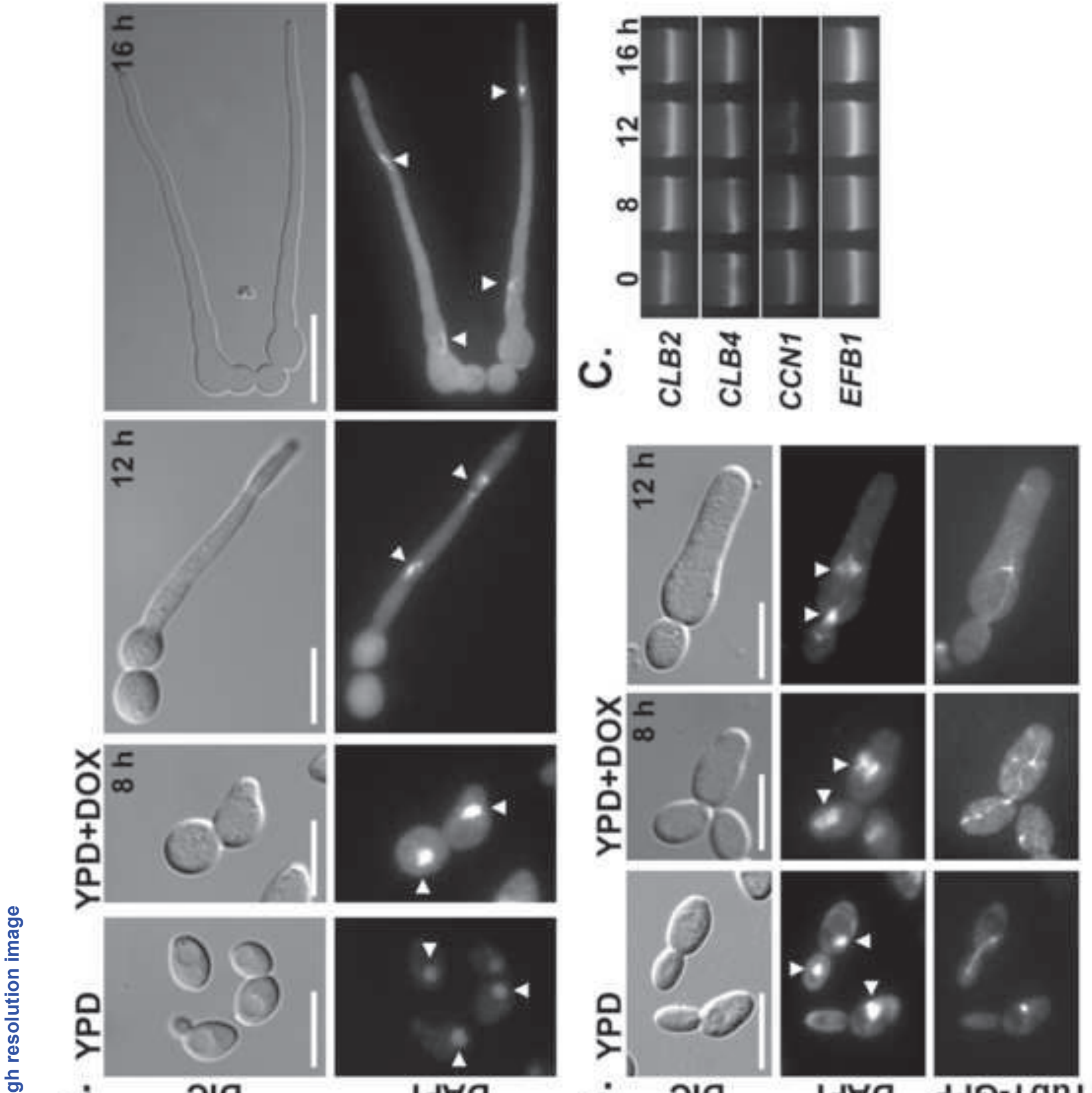

ष ग0

Id $\forall 0$

m

$I d \forall 0$ d $\exists \partial-\left\llcorner q n_{\perp}\right.$

$\wedge$

宁

it 


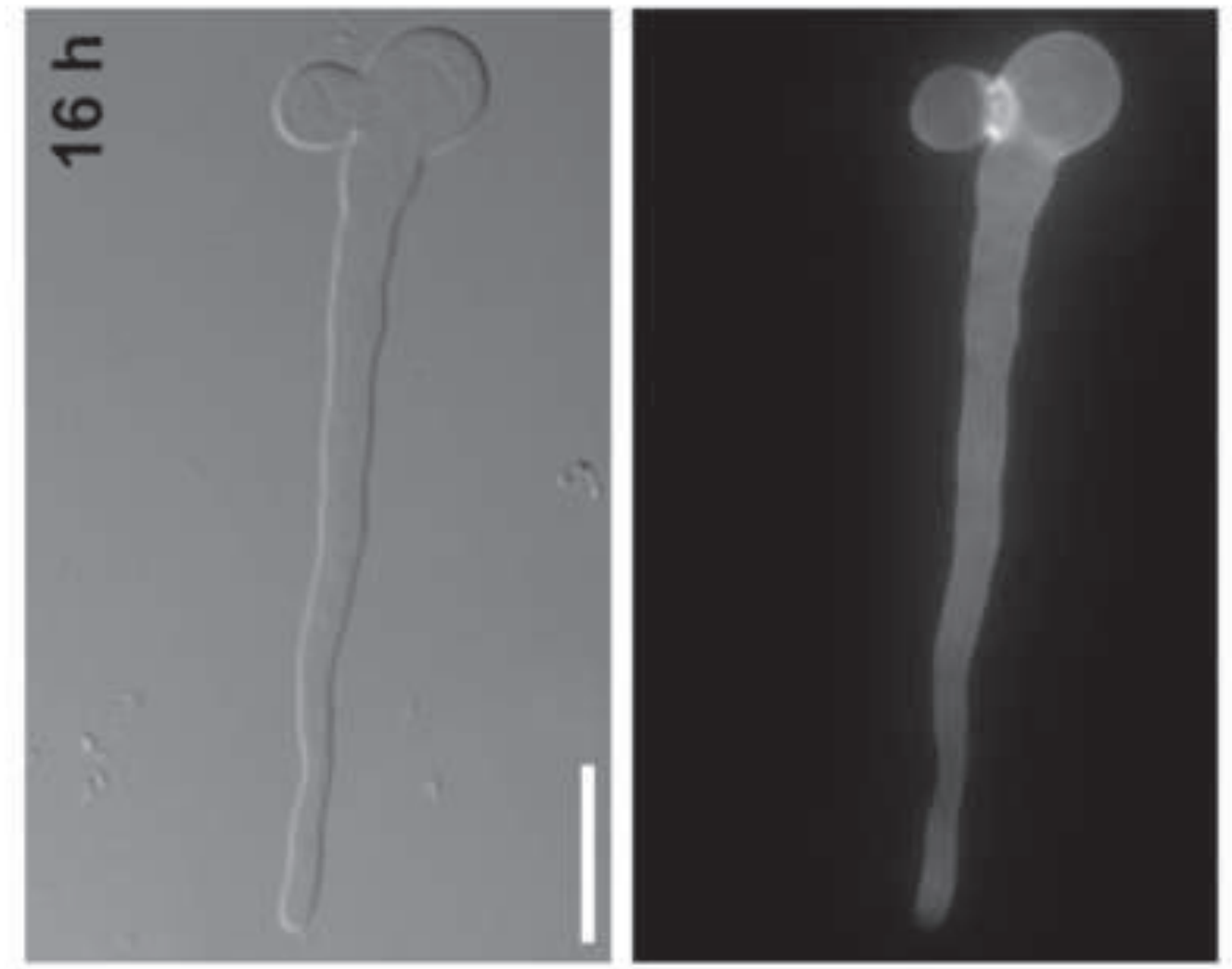

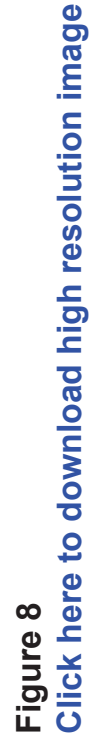

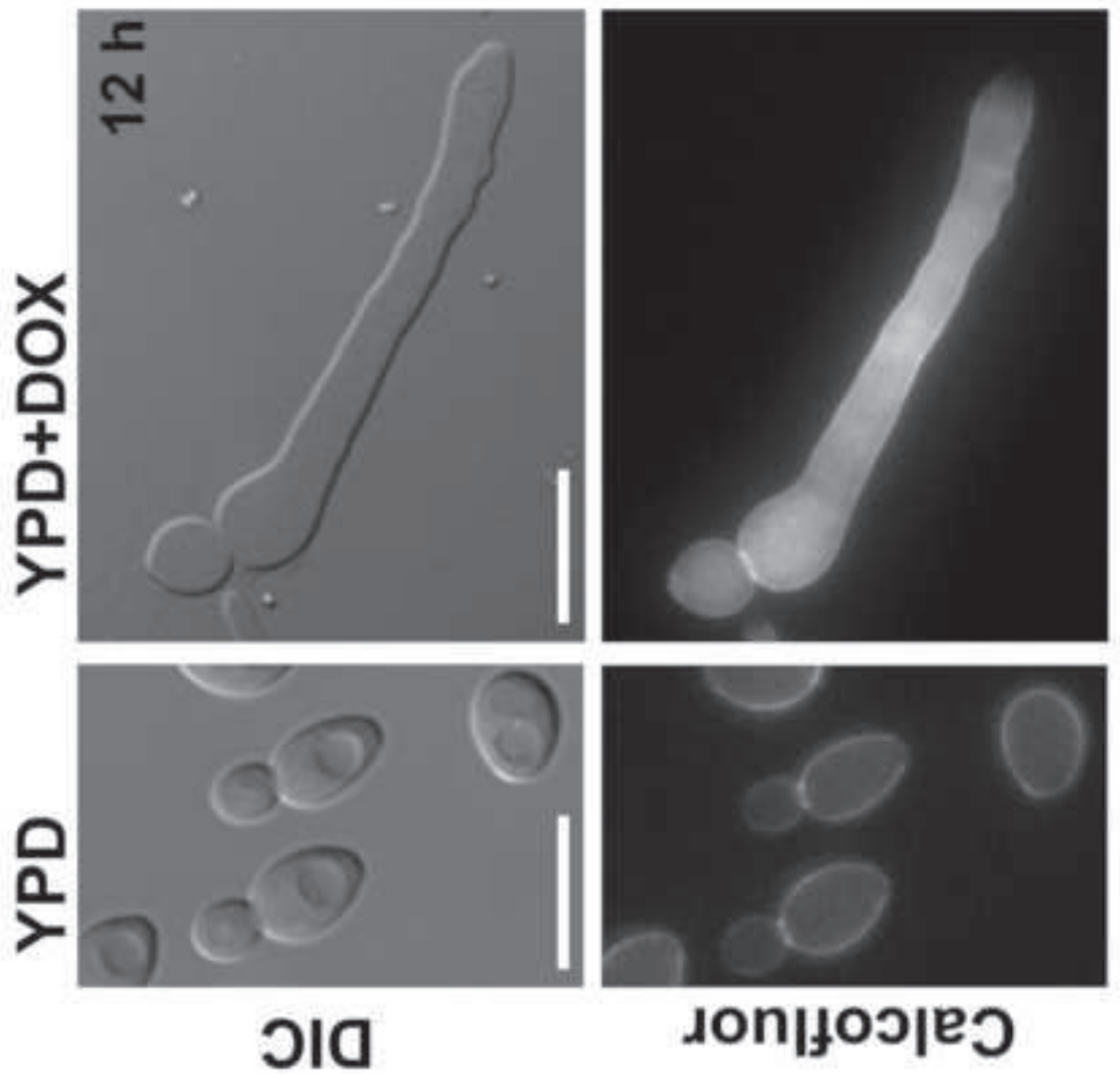

$\infty$ 

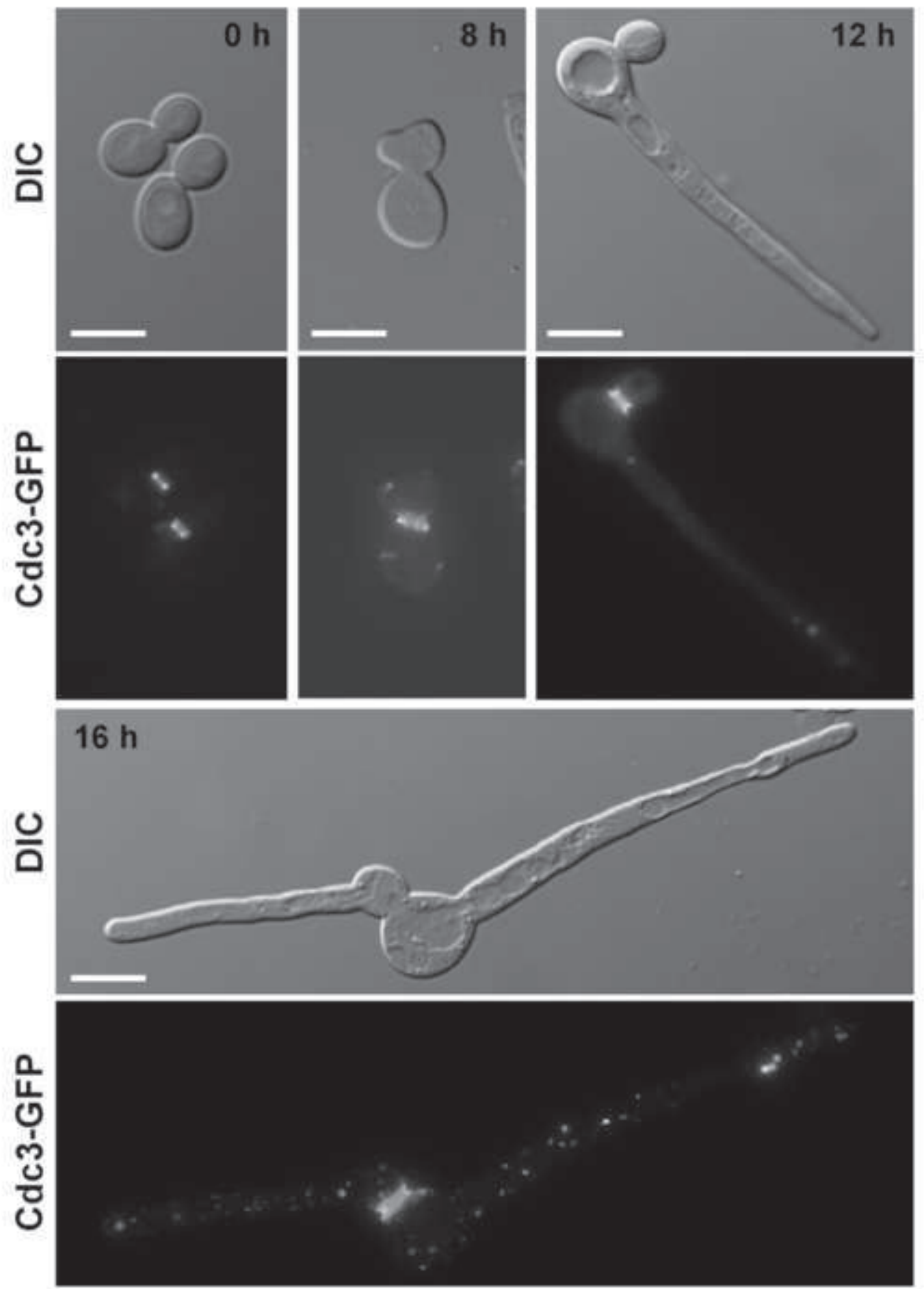